Daniel Hernández Ruipérez · Ana Cristina López Martín

- Fernando Sancho de Salas

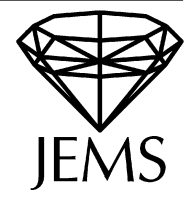

\title{
Relative integral functors for singular fibrations and singular partners
}

Received November 21, 2006 and in revised form July 7, 2007

\begin{abstract}
We study relative integral functors for singular schemes and characterise those which preserve boundedness and those which have integral right adjoints. We prove that a relative integral functor is an equivalence if and only if its restriction to every fibre is an equivalence. This allows us to construct a non-trivial auto-equivalence of the derived category of an arbitrary genus one fibration with no conditions on either the base or the total space and getting rid of the usual assumption of irreducibility of the fibres. We also extend to Cohen-Macaulay schemes the criterion of Bondal and Orlov for an integral functor to be fully faithful in characteristic zero and give a different criterion which is valid in arbitrary characteristic. Finally, we prove that for projective schemes both the Cohen-Macaulay and the Gorenstein conditions are invariant under Fourier-Mukai functors.
\end{abstract}

Keywords. Geometric integral functors, Fourier-Mukai, Cohen-Macaulay, fully faithful, elliptic fibration, equivalence of categories

\section{Contents}

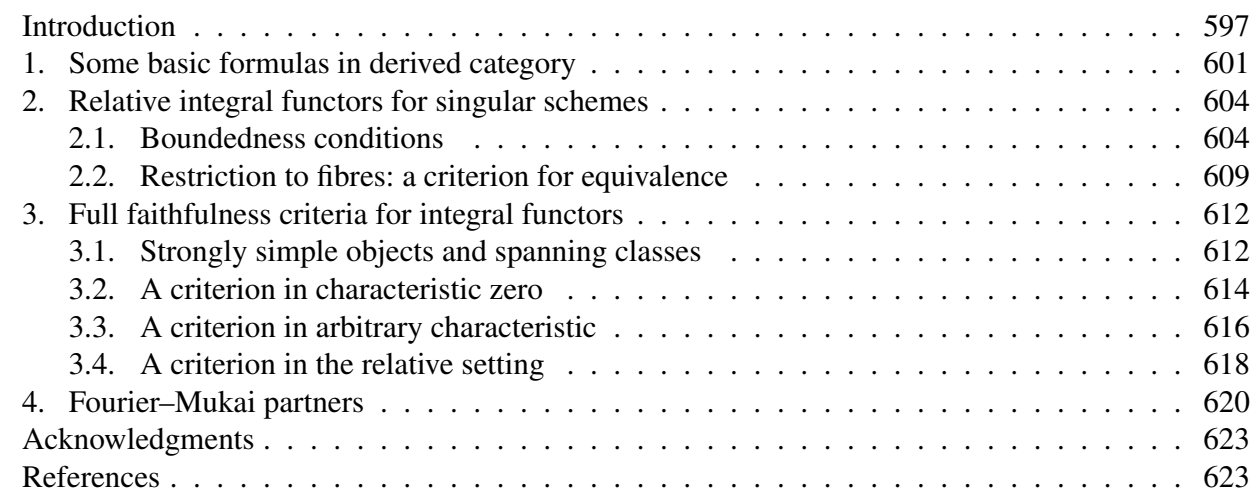

D. Hernández Ruipérez, A. C. López Martín, F. Sancho de Salas: Departamento de Matemáticas and Instituto Universitario de Física Fundamental y Matemáticas (IUFFYM), Universidad de Salamanca, Plaza de la Merced 1-4, 37008 Salamanca, Spain; e-mail: ruiperez@usal.es, anacris@usal.es, fsancho@usal.es

Mathematics Subject Classification (2000): Primary 18E30; Secondary 14F05, 14J27, 14E30, 13D22, 14M05 


\section{Introduction}

The relevance of derived categories and Fourier-Mukai functors in birational geometry is nowadays well known [8, 18]. One of the most important problems in this context is the minimal model problem. There are examples proving that derived categories have a nice behavior under some birational transformations as blow-ups, flips and flops. Furthermore, Bondal and Orlov conjectured [5] that each flip (resp. flop) relating two smooth varieties $X$ and $X^{+}$should induce a fully faithful functor (resp. an equivalence) from the bounded derived category of coherent sheaves on $X^{+}, D_{c}^{b}\left(X^{+}\right)$, to the corresponding bounded derived category $D_{c}^{b}(X)$. Since varieties with singularities have to be allowed in the minimal model programme, one of the main problems this programme leads to is the study of derived categories for singular projective varieties. However, not too much attention has been paid to singular varieties in the literature on the topic. The reason may be that many of the fundamental results rely deeply on smoothness.

In dimension three, the difficulties arising when one deals with Gorenstein schemes can be circumvented using a smoothing approach [13]. Threefold Gorenstein terminal singularities are isolated hypersurface singularities [21] and a hypersurface singularity can be considered as a special fibre of a smooth fourfold. Thus, one can get some information about the derived categories in dimension 3 from the derived categories of the corresponding smooth fourfold. The results in [13] can be generalized to $Q$-Gorenstein teminal threefolds (see [1, 18, 19]). In this situation, the associated Gorenstein stack allows us to reduce the problem to the Gorenstein case. Nevertheless, since in higher dimension there are not local models for quotient singularities and these seem to be more rigid, this smoothing idea does not work and new results are needed.

Following a completely different path, we started in [16] the study of derived categories and fully faithful integral functors for schemes with Gorenstein singularities or fibred in schemes of that kind, and generalised to that situation the characterisation of full faithfulness originally proved by Bondal and Orlov [6] in the smooth case.

The aim of this paper is twofold. On the one hand, we give a very general result that characterizes when relative integral functors are equivalences and that allows us to reduce the problem to the absolute setting. This gives in particular a construction of a non-trivial invertible integral functor for a general genus one fibration. Notice that we adopt here a slightly different definition of a genus one fibration; actually, we mean a flat Gorenstein morphism whose fibres are curves of arithmetic genus 1 and have trivial dualising sheaf; the last condition is a consequence of the others when the fibres are reduced. In the case of dimensions two or three, we are then allowing all types of Kodaira fibre, thus getting rid of the usual assumption of irreducible fibres.

Moreover, for full faithfulness we are now able to consider more general singularities, namely Cohen-Macaulay schemes, both in the absolute and in the relative case. The case of positive characteristic, never studied before, is considered as well. On the other hand, we also tackle the question of what geometric information on the scheme can be recovered from its derived category. For projective schemes, we give an affirmative answer for both the Cohen-Macaulay and the Gorenstein properties.

The paper is organised as follows. 
Section 1 is a repository of formulas used throughout the work and collected here for the reader's convenience.

In Section 2 we study integral functors, which we define directly in the relative situation, and determine conditions for them to map bounded complexes to bounded complexes and to have right adjoints with the same property. The study requires the notions of complexes of relative finite projective dimension and of relative finite homological dimension, which are equivalent in the case of projective morphisms as we proved in [16]. Among the results in Section 2 we can mention Proposition 2.10, where we prove that if the integral functor defined by a kernel $\mathcal{K}^{\bullet} \in D_{c}^{b}(X \times Y)$ induces an equivalence between the bounded derived categories of $X$ and $Y$, then the kernel has to be of finite homological dimension over both $X$ and $Y$, and the same holds for the kernel $\mathbf{R} \mathcal{H}_{o m^{\circ}} \dot{\mathcal{O}}_{X \times Y}\left(\mathcal{K} \bullet, \pi_{Y}^{!} \mathcal{O}_{Y}\right)$ of the right adjoint.

The main result of this section is, however, Proposition 2.15 that proves, in great generality, that a relative integral functor is fully faithful (or an equivalence) if and only if the absolute integral functors induced on the fibres are fully faithful (or equivalences). This result could be useful for the study of relative Fourier-Mukai transforms between the derived categories of the total spaces of two very general fibrations. The reason is that it allows one to pass from a relative to an absolute situation where more is known. For instance, in the absolute setting, there is an important class of well-known Fourier-Mukai functors: the twist functors along spherical objects that were first introduced by Seidel and Thomas in [26]. As a direct corollary of this proposition, we construct a non-trivial auto-equivalence of the derived category of an arbitrary genus one fibration. The result is the following.

Theorem (Proposition 2.16). Let $S$ be an algebraic scheme, $X \rightarrow S$ a genus one fibration, and $\mathcal{I}_{\Delta}$ the ideal sheaf of the relative diagonal. The relative integral functor

$$
\Phi_{X \rightarrow X}^{\mathcal{I}_{\Delta}}: D_{c}^{b}(X) \rightarrow D_{c}^{b}(X)
$$

is an equivalence of categories.

This extends the result and the proof given in [16, Prop. 2.7] in two directions. First the characteristic of the base field is arbitrary. Second, all kinds of possible fibres of a genus one fibration are allowed; in particular, for dimensions two or three they are all Kodaira fibres. Moreover, no condition is imposed on either the base $S$ or the total space $X$ of the fibration.

When the fibration has only integral fibres, Proposition 2.16 gives a short proof of the invertibility of the usual elliptic integral functor. We expect that Proposition 2.16 could be a useful tool for the study of the moduli spaces of relatively semistable sheaves on $X \rightarrow S$ with respect to a suitable relative ample divisor following [3, 2, 9, 15], and for the study of the derived category of $X$ generalising [10].

In Section 3 we give criteria to characterise fully faithful integral functors. We first consider the case of characteristic zero, and extend to Cohen-Macaulay schemes the characterisation of fully faithful integral functors given by Bondal and Orlov. The characterisation result is the following. 
Theorem (Theorem 3.6). Let $X$ and $Y$ be proper schemes over an algebraically closed field of characteristic zero, and let $\mathcal{K} \bullet$ be an object in $D_{c}^{b}(X \times Y)$ of finite homological dimension over both $X$ and $Y$. Assume also that $X$ is projective, Cohen-Macaulay and integral. Then the functor $\Phi_{X \rightarrow Y}^{\mathcal{K}^{\bullet}}: D_{c}^{b}(X) \rightarrow D_{c}^{b}(Y)$ is fully faithful if and only if the kernel $\mathcal{K} \bullet$ is strongly simple over $X$.

This theorem also generalises the extension to varieties with Gorenstein singularities given in [16, Thm. 1.22]. The new theorem is also stronger in the Gorenstein case, because we do not need to assume any longer that $Y$ is projective and Gorenstein.

As in the Gorenstein case, strong simplicity in the Cohen-Macaulay case (Definition 3.3 is defined in terms of locally complete intersection zero cycles instead of the structure sheaves of the closed points. In the smooth case, our definition is weaker that the usual one given by Bondal and Orlov, and Theorem 3.6 improves the characterization of full faithfulness of Bondal and Orlov. It should be noticed that we give here a slightly different notion of strong simplicity than the one given in [16] for Gorenstein varieties. Since both characterise the full faithfulness of the corresponding integral functors, they are equivalent in the Gorenstein case.

We also consider the case of positive characteristic, which is different because Theorem 3.6 fails to hold in that situation. We have modified the notion of strong simplicity to a new one, which also characterises full faithfulness in arbitrary characteristic. The precise statement is:

Theorem (Theorem 3.8. Let $X$ and $Y$ be proper schemes over an algebraically closed field of arbitrary characteristic, and let $\mathcal{K} \bullet$ be an object in $D_{c}^{b}(X \times Y)$ of finite homological dimension over both $X$ and $Y$. Assume also that $X$ is connected, equidimensional, projective and Cohen-Macaulay. Then the functor $\Phi_{X \rightarrow Y}^{\mathcal{K}^{\bullet}}: D_{c}^{b}(X) \rightarrow D_{c}^{b}(Y)$ is fully faithful if and only if the kernel $\mathcal{K} \cdot$ has the following properties:

(1) For every closed point $x \in X$ there is a l.c.i. zero cycle $Z_{x}$ supported on $x$ such that

$$
\operatorname{Hom}_{D(Y)}^{i}\left(\Phi_{X \rightarrow Y}^{\mathcal{K}^{\bullet}}\left(\mathcal{O}_{Z_{x_{1}}}\right), \Phi_{X \rightarrow Y}^{\mathcal{K}^{\bullet}}\left(\mathcal{O}_{x_{2}}\right)\right)=0
$$

unless $x_{1}=x_{2}$ and $0 \leq i \leq \operatorname{dim} X$.

(2) There exists a closed point $x$ such that at least one of the following conditions is satisfied:

(2.1) $\operatorname{Hom}_{D(Y)}^{0}\left(\Phi_{X \rightarrow Y}^{\mathcal{K} \bullet}\left(\mathcal{O}_{X}\right), \Phi_{X \rightarrow Y}^{\mathcal{K} \bullet}\left(\mathcal{O}_{X}\right)\right) \simeq k$.

(2.2) $\operatorname{Hom}_{D(Y)}^{0}\left(\Phi_{X \rightarrow Y}^{\mathcal{K} \bullet}\left(\mathcal{O}_{Z_{x}}\right), \Phi_{X \rightarrow Y}^{\mathcal{K}^{\bullet}}\left(\mathcal{O}_{x}\right)\right) \simeq k$ for any l.c.i. zero cycle $Z_{x}$ supported on $x$.

(2.3) $\operatorname{dim}_{k} \operatorname{Hom}_{D(Y)}^{0}\left(\Phi_{X \rightarrow Y}^{\mathcal{K} \bullet}\left(\mathcal{O}_{Z_{x}}\right), \Phi_{X \rightarrow Y}^{\mathcal{K} \bullet}\left(\mathcal{O}_{Z_{x}}\right)\right) \leq l\left(\mathcal{O}_{Z_{x}}\right)$ for any l.c.i. zero cycle $Z_{x}$ supported on $x$, where $l\left(\mathcal{O}_{Z_{x}}\right)$ is the length of $\mathcal{O}_{Z_{x}}$.

Due to Proposition 2.15 and the properties of relative integral functors proved in Section 2, the extension of the above criteria to the relative setting is straightforward (see Theorem 3.11).

As an application we give a different proof of Proposition 2.16 which does not need to use the work of Seidel and Thomas about spherical objects. 
The last Section 4 is devoted to the study of Fourier-Mukai partners of a given proper scheme $X$, that is, proper schemes $Y$ with equivalent coherent bounded category (i.e., $D$-equivalent to $X$ ), and such that the equivalence is given by an integral functor. In the projective smooth case, the second condition is automatically satisfied, due to Orlov's representation theorem [25]. However, the validity of Orlov's theorem for singular varieties is still unknown; hence, in principle, two $D$-equivalent singular varieties might not be Fourier-Mukai partners.

It is known that smooth Fourier-Mukai partners share many geometrical properties. In the same vein, we prove in Section 4 that singular Fourier-Mukai partners also have many geometrical properties in common. Our main result in this direction is the following.

Theorem (Theorem 4.4). Let $X$ be a projective equidimensional Cohen-Macaulay scheme and $Y$ a projective Fourier-Mukai partner of $X$.

(1) If $Y$ is reduced, then $Y$ is equidimensional and $\operatorname{dim} Y=\operatorname{dim} X$.

(2) If $Y$ is equidimensional and $\operatorname{dim} Y=\operatorname{dim} X$, then $Y$ is Cohen-Macaulay. Moreover, if $X$ is Gorenstein, then $Y$ is Gorenstein as well.

Conventions. In this paper, scheme means separated scheme of finite type over an algebraically closed field $k$. By a Gorenstein or a Cohen-Macaulay morphism, we understand a flat morphism of schemes whose fibres are respectively Gorenstein or Cohen-Macaulay. For any scheme $X$ we denote by $D(X)$ the derived category of complexes of $\mathcal{O}_{X}$-modules with quasi-coherent cohomology sheaves. This is the essential image of the derived category of quasi-coherent sheaves in the derived category $D(\mathfrak{M o d}(X))$ of all $\mathcal{O}_{X}$-modules [4, Cor. 5.5]. Analogously, $D^{+}(X), D^{-}(X)$ and $D^{b}(X)$ will denote the derived categories of complexes which are respectively bounded below, bounded above and bounded on both sides, and have quasi-coherent cohomology sheaves. The subscript $c$ will refer to the corresponding subcategories of complexes with coherent cohomology sheaves.

\section{Some basic formulas in derived category}

We recall here some basic formulas which will be used in the rest of the paper.

If $X$ is a scheme, there is a functorial isomorphism (in the derived category)

$$
\mathbf{R H o m}_{\mathcal{O}_{X}}\left(\mathcal{F}^{\bullet}, \mathbf{R} \mathcal{H o m}_{\mathcal{O}_{X}}^{\bullet}\left(\mathcal{E}^{\bullet}, \mathcal{H}^{\bullet}\right)\right) \stackrel{\sim}{\rightarrow} \mathbf{R} \mathcal{H o m}_{\mathcal{O}_{X}}\left(\mathcal{F}^{\bullet} \stackrel{\mathbf{L}}{\otimes} \mathcal{E}^{\bullet}, \mathcal{H}^{\bullet}\right)
$$

where $\mathcal{F}^{\bullet}, \mathcal{E}^{\bullet}$ and $\mathcal{H}^{\bullet}$ are in $D(\mathfrak{M o d}(X))$ [27, Thm. A]. One also has a functorial isomorphism in $D(\mathfrak{M o d}(X))$

$$
\mathbf{R} \mathcal{H o m}_{\mathcal{O}_{X}}^{\bullet}\left(\mathcal{F}^{\bullet}, \mathcal{E}^{\bullet}\right) \stackrel{\mathbf{L}}{\otimes} \mathcal{H}^{\bullet} \stackrel{\sim}{\rightarrow} \mathbf{R} \mathcal{H} \operatorname{Hom}_{\mathcal{O}_{X}}\left(\mathcal{F}^{\bullet}, \mathcal{E}^{\bullet} \stackrel{\mathbf{L}}{\otimes} \mathcal{H}^{\bullet}\right)
$$

when either $\mathcal{F}^{\bullet}$ or $\mathcal{H}^{\bullet}$ has finite homological dimension. When $\mathcal{F}^{\bullet}$ is bounded above with coherent cohomology sheaves, while $\mathcal{E}^{\bullet}$ is bounded below and $\mathcal{H}^{\bullet}$ has finite homological dimension, the formula is standard (cf. [14, Prop. II.5.14]). Since we have not found a 
reference for the unbounded case, we give a simple proof here: Let $\mathcal{E}^{\bullet} \rightarrow \mathcal{I}^{\bullet}$ be an injective resolution [27, Thm. 4.5] and $\mathcal{P}^{\bullet} \rightarrow \mathcal{H}^{\bullet}$ a flat resolution [27, Prop. 5.6]. One has morphisms of complexes

$$
\operatorname{Hom}_{\mathcal{O}_{X}}\left(\mathcal{F}^{\bullet}, \mathcal{I}^{\bullet}\right) \otimes \mathcal{P}^{\bullet} \rightarrow \mathcal{H o m}_{\mathcal{O}_{X}}^{\bullet}\left(\mathcal{F}^{\bullet}, \mathcal{I}^{\bullet} \otimes \mathcal{P}^{\bullet}\right) \rightarrow \mathcal{H o m}_{\mathcal{O}_{X}}\left(\mathcal{F}^{\bullet}, \mathcal{J}^{\bullet}\right)
$$

where $\mathcal{J}^{\bullet}$ is an injective resolution of $\mathcal{I} \bullet \otimes \mathcal{P}^{\bullet}$. This proves the existence of a morphism

$$
\mathbf{R} \mathcal{H o m}_{\mathcal{O}_{X}}\left(\mathcal{F}^{\bullet}, \mathcal{E}^{\bullet}\right) \stackrel{\mathbf{L}}{\otimes} \mathcal{H}^{\bullet} \rightarrow \mathbf{R} \mathcal{H o m}_{\mathcal{O}_{X}}\left(\mathcal{F}^{\bullet}, \mathcal{E}^{\bullet} \stackrel{\mathbf{L}}{\otimes} \mathcal{H}^{\bullet}\right)
$$

We now prove that this is an isomorphism if either $\mathcal{F} \bullet$ or $\mathcal{H}^{\bullet}$ has finite homological dimension. This is a local question, so we may assume that $X=\operatorname{Spec} A$ is affine and that either $\mathcal{F}^{\bullet}$ or $\mathcal{P}^{\bullet}$ is a bounded complex of free $A$-modules of finite rank. In both cases we have an isomorphism of complexes

$$
\mathcal{H o m}_{A}^{\bullet}\left(\mathcal{F}^{\bullet}, \mathcal{I}^{\bullet}\right) \otimes \mathcal{P}^{\bullet} \stackrel{\sim}{\rightarrow} \mathcal{H o m}_{A}^{\bullet}\left(\mathcal{F}^{\bullet}, \mathcal{I}^{\bullet} \otimes \mathcal{P}^{\bullet}\right) .
$$

In the first case, this proves the isomorphism (1.2) directly. In the second case, we have only to take into account that since $\mathcal{P}^{\bullet}$ is a bounded complex of free modules of finite rank, the complex $\mathcal{I} \bullet \otimes \mathcal{P}^{\bullet}$ is injective.

If $f: X \rightarrow Y$ is a morphism of schemes, the direct and inverse images are defined for unbounded complexes, $\mathbf{R} f_{*}: D(\mathfrak{M o d}(X)) \rightarrow D(\mathfrak{M o d}(Y)), \mathbf{L} f^{*}: D(\mathfrak{M o d}(Y)) \rightarrow$ $D\left(\mathfrak{M}_{\mathfrak{o}}(X)\right)$ and the latter is a right adjoint to the former [27, Thm. B]. They induce morphisms $\mathbf{R} f_{*}: D(X) \rightarrow D(Y), \mathbf{L} f^{*}: D(Y) \rightarrow D(X)$ which are adjoint to each other as well. One has a "projection formula"

$$
\mathbf{R} f_{*}\left(\mathcal{F} \cdot \stackrel{\mathbf{L}}{\otimes} \mathbf{L} f^{*} \mathcal{G} \cdot\right) \simeq \mathbf{R} f_{*} \mathcal{F} \cdot \stackrel{\mathbf{L}}{\otimes} \mathcal{G}^{\bullet}
$$

for $\mathcal{F}^{\bullet}$ in $D(X)$ and $\mathcal{G}^{\bullet}$ in $D(Y)$ [24, Prop. 5.3].

Let us consider a cartesian diagram of morphisms of algebraic varieties

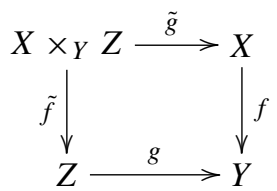

Then for any complex $\mathcal{G} \bullet$ of $\mathcal{O}_{X}$-modules there is a natural morphism

$$
\mathbf{L} g^{*} \mathbf{R} f_{*} \mathcal{G}^{\bullet} \rightarrow \mathbf{R} \tilde{f}_{*} \mathbf{L} \tilde{g}^{*} \mathcal{G}^{\bullet} .
$$

Moreover, if $\mathcal{G}^{\bullet}$ is in $D(X)$ and either $f$ or $g$ is flat, the above morphism is an isomorphism. This is the so-called "base-change formula" in the derived category. The flat basechange formula, i.e., when $g$ is flat, is well-known [14, Prop. II.5.12]. If $g$ is arbitrary and $f$ is flat, the formula is proven in [2, Appendix A]. In this paper we only need the following very simple case. 
Proposition 1.1. Let us consider a diagram

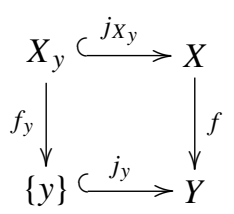

where $f$ is a flat morphism of schemes, $y \in Y$ is a closed point and $X_{y}=f^{-1}(y)$ is the fibre. For every object $\mathcal{G} \bullet$ in $D(X)$, there is a base-change isomorphism

$$
\mathbf{L} j_{y}^{*} \mathbf{R} f_{*} \mathcal{G} \cdot \simeq \mathbf{R} f_{y *} \mathbf{L} j_{X_{y}}^{*} \mathcal{G} \cdot
$$

in the derived category.

Proof. It is enough to prove that the induced morphism

$$
j_{y *}\left(\mathbf{L} j_{y}^{*} \mathbf{R} f_{*} \mathcal{G}^{\bullet}\right) \rightarrow j_{y *}\left(\mathbf{R} f_{y *} \mathbf{L} j_{X_{y}}^{*} \mathcal{G}^{\bullet}\right)
$$

is an isomorphism in $D(Y)$. By the projection formula, the left member is isomorphic to $\mathcal{O}_{y} \stackrel{\mathbf{L}}{\otimes} \mathbf{R} f_{*} \mathcal{G}^{\bullet}$. The right member is isomorphic to $\mathbf{R} f_{*} j_{X_{y} *} \mathbf{L} j_{X_{y}}^{*} \mathcal{G}^{\bullet}$, which is isomorphic to $\mathbf{R} f_{*}\left(\mathcal{O}_{X_{y}} \stackrel{\mathbf{L}}{\otimes} \mathcal{G}^{\bullet}\right)$ again by the projection formula. Moreover, $\mathcal{O}_{X_{y}} \simeq \mathbf{L} f^{*} \mathcal{O}_{y}$ because $f$ is flat, and so $\mathbf{R} f_{*}\left(\mathcal{O}_{X_{y}} \stackrel{\mathbf{L}}{\otimes} \mathcal{G}^{\bullet}\right) \simeq \mathcal{O}_{y} \stackrel{\mathbf{L}}{\otimes} \mathbf{R} f_{*} \mathcal{G}^{\bullet}$ by the projection formula for $f$.

Let $f: X \rightarrow Y$ be a proper morphism of schemes. The relative Grothendieck duality states the existence of a functorial isomorphism in the derived category

$$
\mathbf{R} \mathcal{H o m}_{\mathcal{O}_{Y}}^{\cdot}\left(\mathbf{R} f_{*} \mathcal{F}^{\bullet}, \mathcal{G}^{\bullet}\right) \simeq \mathbf{R} f_{*} \mathbf{R} \mathcal{H o m}_{\mathcal{O}_{X}}\left(\mathcal{F}^{\bullet}, f^{!} \mathcal{G}^{\bullet}\right)
$$

for $\mathcal{G}^{\bullet}$ in $D(Y)$ and $\mathcal{F}^{\bullet}$ in $D(X)$ (see for instance [24]). By applying the derived functor of the global section functor, we obtain the global duality formula

$$
\operatorname{Hom}_{D(Y)}\left(\mathbf{R} f_{*} \mathcal{F}^{\bullet}, \mathcal{G}^{\bullet}\right) \simeq \operatorname{Hom}_{D(X)}\left(\mathcal{F}^{\bullet}, f^{!} \mathcal{G}^{\bullet}\right) .
$$

In other words, the direct image $\mathbf{R} f_{*}: D(X) \rightarrow D(Y)$ has a right adjoint $f^{!}: D(Y) \rightarrow$ $D(X)$. If $g: Y \rightarrow Z$ is another proper morphism, there is a natural functor isomorphism $(g \circ f)^{!} \simeq f^{!} \circ g^{!}$.

We shall call the object $f^{!} \mathcal{O}_{Y}$ the relative dualising complex of $X$ over $Y$. When $Y$ is a point, we also write $\mathcal{D}_{X}^{\bullet}$ instead of $f^{!} \mathcal{O}_{Y}$.

When $f$ is flat, it is Cohen-Macaulay if and only if the relative dualising complex $f^{!} \mathcal{O}_{Y}$ is isomorphic to a single sheaf $\omega_{X / Y}$ placed in degree $-n$ (where $n$ is the relative dimension of $f$ ). Moreover, $\omega_{X / Y}$ is a line bundle if and only if $f$ is a Gorenstein morphism.

We finish this part by recalling some properties of Grothendieck duality which we shall use in this paper. 
First, if $f: X \rightarrow Y$ is a finite morphism, then $f_{*} f^{!} \mathcal{G} \bullet \mathbf{R} \mathcal{H o m}_{\mathcal{O}_{Y}}\left(f_{*} \mathcal{O}_{X}, \mathcal{G}^{\bullet}\right)$. Secondly, Grothendieck duality is compatible with flat base-change, that is, if $g: Z \rightarrow Y$ is a flat morphism and $f_{Z}: Z \times_{Y} X \rightarrow Z$ is the induced morphism, then $f_{Z}^{!} \mathcal{O}_{Z} \simeq$ $g_{X}^{*} f^{!} \mathcal{O}_{Y}$ where $g_{X}: Z \times_{Y} X \rightarrow X$ is the projection. In particular, the formation of $f^{!} \mathcal{O}_{Y}$ is compatible with open immersions $U \subseteq Y$. Finally, there is a natural map $\mathbf{L} f^{*} \mathcal{G} \bullet \stackrel{\mathbf{L}}{\otimes} f^{!} \mathcal{O}_{Y} \rightarrow f^{!} \mathcal{G} \bullet$; in some cases, it is an isomorphism. One of those cases is when $\mathcal{G}^{\bullet}$ has finite homological dimension; to see this, one has to prove that for any complex $\mathcal{F}^{\bullet}$ in $D(X)$ the induced map $\operatorname{Hom}_{D(X)}\left(\mathcal{F}^{\bullet}, \mathbf{L} f^{*} \mathcal{G}^{\bullet} \stackrel{\mathbf{L}}{\otimes} f^{!} \mathcal{O}_{Y}\right) \rightarrow \operatorname{Hom}_{D(X)}\left(\mathcal{F}^{\bullet}, f^{!} \mathcal{G}^{\bullet}\right)$ is bijective, and for this it is enough to prove that $\mathbf{R} f_{*} \mathbf{R} \mathcal{H} \operatorname{Hom}_{\mathcal{O}_{X}}\left(\mathcal{F}^{\bullet}, \mathbf{L} f^{*} \mathcal{G} \bullet \stackrel{\mathbf{L}}{\otimes} f^{!} \mathcal{O}_{Y}\right) \rightarrow$ $\mathbf{R} f_{*} \mathbf{R} \mathcal{H}^{\circ}{ }_{\mathcal{O}_{X}}\left(\mathcal{F}^{\bullet}, f^{!} \mathcal{G}^{\bullet}\right)$ is an isomorphism in $D(Y)$. The left member is isomorphic to $\mathbf{R} \mathcal{H}_{o m^{\bullet}} \mathcal{O}_{Y}\left(\mathbf{R} f_{*} \mathcal{F}^{\bullet}, \mathcal{O}_{Y}\right) \stackrel{\mathbf{L}}{\otimes} \mathcal{G}^{\bullet}$, by $[1.2$, the projection formula and the duality isomorphism 1.3. Moreover, $\mathbf{R} \mathcal{H o m}_{\mathcal{O}_{Y}}\left(\mathbf{R} f_{*} \mathcal{F}^{\bullet}, \mathcal{O}_{Y}\right) \stackrel{\mathbf{L}}{\otimes} \mathcal{G} \bullet \simeq \mathbf{R} f_{*} \mathbf{R} \mathcal{H o m}_{\mathcal{O}_{X}}\left(\mathcal{F}^{\bullet}, f^{!} \mathcal{G}^{\bullet}\right)$ by (1.2) and relative duality (1.3). Another case where $\mathbf{L} f^{*} \mathcal{G} \bullet \stackrel{\mathbf{L}}{\otimes} f^{!} \mathcal{O}_{Y} \rightarrow f^{!} \mathcal{G} \bullet$ is an isomorphism is when $f$ is a regular closed immersion; in this case one has to prove that the induced morphism $f_{*}\left(\mathbf{L} f^{*} \mathcal{G}^{\bullet} \stackrel{\mathbf{L}}{\otimes} f^{!} \mathcal{O}_{Y}\right) \rightarrow f_{*}\left(f^{!} \mathcal{G}^{\bullet}\right)$ is an isomorphism. By the projection formula the left member is isomorphic to $\mathcal{G}^{\bullet} \otimes f_{*}\left(f^{!} \mathcal{O}_{Y}\right)$. Since $f_{*} f^{!} \mathcal{O}_{Y} \simeq$ $\mathbf{R} \mathcal{H}^{\circ} \dot{\mathcal{O}}_{Y}\left(f_{*} \mathcal{O}_{X}, \mathcal{O}_{Y}\right)$ and $f_{*} f^{!} \mathcal{G}^{\bullet} \simeq \mathbf{R} \mathcal{H o m}_{\mathcal{O}_{Y}}^{\bullet}\left(f_{*} \mathcal{O}_{X}, \mathcal{G}^{\bullet}\right)$, the result follows again from (1.2), which can be applied because $f_{*} \mathcal{O}_{X}$ is of finite homological dimension.

\section{Relative integral functors for singular schemes}

\subsection{Boundedness conditions}

Let $S$ be a scheme and let $X \rightarrow S$ and $Y \rightarrow S$ be proper morphisms. We denote by $\pi_{X}$ and $\pi_{Y}$ the projections of the fibre product $X \times{ }_{S} Y$ onto its factors.

Let $\mathcal{K} \bullet$ be an object in $D_{c}^{b}\left(X \times_{S} Y\right)$. The relative integral functor defined by $\mathcal{K}^{\bullet}$ is the functor $\Phi_{X \rightarrow Y}^{\mathcal{K}^{\bullet}}: D(X) \rightarrow D(Y)$ given by

$$
\Phi_{X \rightarrow Y}^{\mathcal{K}}\left(\mathcal{F}^{\bullet}\right)=\mathbf{R} \pi_{Y *}\left(\mathbf{L} \pi_{X}^{*} \mathcal{F}^{\bullet} \stackrel{\mathbf{L}}{\otimes} \mathcal{K}^{\bullet}\right)
$$

and it maps $D^{-}(X)$ to $D^{-}(Y)$.

By adjunction between the direct and inverse images and by relative Grothendieck duality, $\Phi_{X \rightarrow Y}^{\mathcal{K}^{\bullet}}$ has a right adjoint $H: D(Y) \rightarrow D(X)$ given by

$$
H\left(\mathcal{G}^{\bullet}\right)=\mathbf{R} \pi_{X, *}\left(\mathbf{R} \mathcal{H}_{o m^{\circ}}^{\bullet} \mathcal{O}_{X \times Y}\left(\mathcal{K}^{\bullet}, \pi_{Y}^{!} \mathcal{G}^{\bullet}\right)\right),
$$

which maps $D^{+}(Y)$ to $D^{+}(X)$. We shall now study when either $\Phi_{X \rightarrow Y}^{\mathcal{K} \bullet}$ or $H$ take bounded complexes to bounded complexes. 
Definition 2.1. Let $f: Z \rightarrow T$ be a morphism of schemes. An object $\mathcal{E}^{\bullet}$ in $D_{c}^{b}(Z)$ is said to be of finite homological dimension (resp. of finite projective dimension) over $T$ if $\mathcal{E} \bullet \stackrel{\mathbf{L}}{\otimes} \mathbf{L} f^{*} \mathcal{G}^{\bullet}\left(\right.$ resp. $\left.\mathbf{R} \mathcal{H o m}_{\mathcal{O}_{X}}\left(\mathcal{E}^{\bullet}, f^{!} \mathcal{G}^{\bullet}\right)\right)$ is bounded for any $\mathcal{G}^{\bullet}$ in $D_{c}^{b}(T)$.

Remark 2.2. The absolute notion of finite homological dimension corresponds to being "of finite homological dimension over $Z$ with respect to the identity" [16, Lemma 1.2], rather than being "of finite homological dimension over Spec $k$ "; actually, any object $\mathcal{E}$ • in $D_{c}^{b}(Z)$ is of finite homological dimension over Spec $k$. The usual notion of morphism of finite homological dimension is equivalent to saying that $\mathcal{O}_{Z}$ is "of finite homological dimension over $T$ ".

For projective morphisms one has the following result (see [16]):

Proposition 2.3. Let $f: Z \rightarrow T$ be a projective morphism and $\mathcal{O}(1)$ a relatively very ample line bundle. Let $\mathcal{E}^{\bullet}$ be an object of $D_{c}^{b}(Z)$ and $\operatorname{set}^{\bullet}(r)=\mathcal{E}^{\bullet} \otimes \mathcal{O}(r)$. The following conditions are equivalent:

(1) $\mathcal{E} \bullet$ is of finite projective dimension over $T$.

(2) $\mathbf{R} f_{*}\left(\mathcal{E}^{\bullet}(r)\right)$ is of finite homological dimension (i.e. a perfect complex) for every integer $r$.

(3) $\mathcal{E}^{\bullet}$ is of finite homological dimension over $T$.

Thus, if $f$ is locally projective, then $\mathcal{E}^{\bullet}$ is of finite projective dimension over $T$ if and only if it is of finite homological dimension over $T$.

Corollary 2.4. Let $f: Z \rightarrow T$ be a locally projective morphism. The functor $f^{!}$sends $D^{b}(T)$ to $D^{b}(Z)$ if and only if $f$ is a morphism of finite homological dimension.

We also state here the following lemma whose proof can be found in [16].

Lemma 2.5. Let $f: Z \rightarrow T$ be a projective morphism.

(1) Let $\mathcal{E}^{\bullet}$ be an object of $D^{-}(Z)$. Then $\mathcal{E}^{\bullet}=0$ (resp. is an object of $D^{b}(Z)$ ) if and only if $\mathbf{R} f_{*}\left(\mathcal{E}^{\bullet}(r)\right)=0$ (resp. is an object of $D^{b}(T)$ ) for every integer $r$.

(2) Let $g: \mathcal{E}^{\bullet} \rightarrow \mathcal{F} \bullet$ be a morphism in $D^{-}(Z)$. Then $g$ is an isomorphism if and only if the induced morphism $\mathbf{R} f_{*}\left(\mathcal{E}^{\bullet}(r)\right) \rightarrow \mathbf{R} f_{*}\left(\mathcal{F}^{\bullet}(r)\right)$ is an isomorphism in $D^{-}(T)$ for every integer $r$.

The arguments used in the proof of the above lemma also show that an object $\mathcal{E}^{\bullet}$ of $D(Z)$ has coherent cohomology sheaves if and only if $\mathbf{R} f_{*}\left(\mathcal{E}^{\bullet}(r)\right)$ has coherent cohomology sheaves for every integer $r$. One then obtains the following result.

Proposition 2.6. If $f: Z \rightarrow T$ is a locally projective morphism, then the functor $f^{!}$ sends $D_{c}(T)$ to $D_{c}(Z)$.

Proof. Let $\mathcal{G}^{\bullet}$ be an object of $D_{c}(T)$. Since the formation of $f^{!} \mathcal{G}^{\bullet}$ is compatible with open immersions, we can assume that $f$ is projective. In this case, Grothendieck duality gives $\mathbf{R} f_{*}\left(f^{!} \mathcal{G}^{\bullet}(r)\right) \simeq \mathbf{R} \mathcal{H o m}_{\mathcal{O}_{Z}}\left(\mathbf{R} f_{*} \mathcal{O}_{Z}(-r), \mathcal{G}^{\bullet}\right)$, and the latter complex has coherent cohomology sheaves. We finish by Lemma 2.5 . 
Proposition 2.7. Assume that $X \rightarrow S$ is locally projective and let $\mathcal{K} \bullet$ be an object in $D_{c}^{b}\left(X \times_{S} Y\right)$. The functor $\Phi_{X \rightarrow Y}^{\mathcal{K}^{\bullet}}$ maps $D_{c}^{b}(X)$ to $D_{c}^{b}(Y)$ if and only if $\mathcal{K} \bullet$ has finite homological dimension over $X$.

Proof. Assume that $\Phi_{X \rightarrow Y}^{\mathcal{K}}$ maps $D_{c}^{b}(X)$ to $D_{c}^{b}(Y)$. We have to prove that $\mathbf{L} \pi_{X}^{*} \mathcal{F}^{\bullet} \otimes \mathcal{K}^{\bullet}$ is bounded for any bounded complex $\mathcal{F}^{\bullet}$. We may assume that $X \rightarrow S$ is projective, and then $\pi_{Y}$ is also projective. By Lemma 2.5, it suffices to show that the complex $\mathbf{R} \pi_{Y *}\left[\mathbf{L} \pi_{X}^{*} \mathcal{F} \bullet \stackrel{\mathbf{L}}{\otimes} \mathcal{K} \bullet \otimes \pi_{X}^{*} \mathcal{O}(r)\right]$ is bounded for any $r$. This is immediate from the equality $\mathbf{R} \pi_{Y *}\left[\mathbf{L} \pi_{X}^{*} \mathcal{F} \bullet \stackrel{\mathbf{L}}{\otimes} \mathcal{K} \bullet \otimes \pi_{X}^{*} \mathcal{O}(r)\right]=\Phi_{X \rightarrow Y}^{\mathcal{K} \bullet}(\mathcal{F} \bullet(r))$. The converse is clear.

Proposition 2.8. Let $f: Z \rightarrow T$ be a locally projective morphism of schemes and $\mathcal{E}^{\bullet}$ an object of $D_{c}^{b}(Z)$ of finite homological dimension over $T$. Then

$$
\mathbf{R} \mathcal{H o m}_{\mathcal{O}_{Z}}\left(\mathcal{E}^{\bullet}, f^{!} \mathcal{O}_{T}\right) \stackrel{\mathbf{L}}{\otimes} \mathbf{L} f^{*} \mathcal{G}^{\bullet} \simeq \mathbf{R} \mathcal{H o m}_{\mathcal{O}_{Z}}\left(\mathcal{E}^{\bullet}, f^{!} \mathcal{G}^{\bullet}\right)
$$

for $\mathcal{G}^{\bullet}$ in $D_{c}^{b}(T)$. In particular, $\mathbf{R} \mathcal{H}_{\text {om }}^{\bullet}{ }_{\mathcal{O}_{Z}}\left(\mathcal{E}^{\bullet}, f^{!} \mathcal{O}_{T}\right)$ is also of finite homological dimension over $T$.

Proof. One has natural morphisms

$$
\begin{aligned}
\mathbf{R H o m}_{\mathcal{O}_{Z}}\left(\mathcal{E}^{\bullet}, f^{!} \mathcal{O}_{T}\right) \stackrel{\mathbf{L}}{\otimes} \mathbf{L} f^{*} \mathcal{G}^{\bullet} \rightarrow \mathbf{R} \mathcal{H o m}_{\mathcal{O}_{Z}}\left(\mathcal{E}^{\bullet}, \mathbf{L} f^{*} \mathcal{G}^{\bullet} \stackrel{\mathbf{L}}{\otimes} f^{!} \mathcal{O}_{T}\right) \\
\rightarrow \mathbf{R} \mathcal{H}{ }^{\bullet} \dot{\mathcal{O}}_{Z}\left(\mathcal{E}^{\bullet}, f^{!} \mathcal{G}^{\bullet}\right)
\end{aligned}
$$

We have to prove that the composition is an isomorphism. This is a local question on $T$, so that we can assume that $f$ is projective.

By Lemma 2.5 we have to prove that the induced morphism

$$
\mathbf{R} f_{*}\left(\mathbf{R} \mathcal{H o m}_{\mathcal{O}_{Z}}\left(\mathcal{E}^{\bullet}, f^{!} \mathcal{O}_{T}\right) \stackrel{\mathbf{L}}{\otimes} \mathbf{L} f^{*} \mathcal{G}^{\bullet} \otimes \mathcal{O}(r)\right) \rightarrow \mathbf{R} f_{*}\left(\mathbf{R} \mathcal{H}_{o m} \bullet_{\mathcal{O}_{Z}}\left(\mathcal{E}^{\bullet}, f^{!} \mathcal{G}^{\bullet}\right) \otimes \mathcal{O}(r)\right)
$$

is an isomorphism for any integer $r$. By Grothendieck duality, this is equivalent to proving that the induced morphism

$$
\mathbf{R} \mathcal{H o m}_{\mathcal{O}_{T}}\left(\mathbf{R} f_{*} \mathcal{E}^{\bullet}(-r), \mathcal{O}_{T}\right) \stackrel{\mathbf{L}}{\otimes} \mathcal{G}^{\bullet} \rightarrow \mathbf{R} \mathcal{H o m}_{\mathcal{O}_{T}}\left(\mathbf{R} f_{*} \mathcal{E}^{\bullet}(-r) \mathcal{G}^{\bullet}\right)
$$

is an isomorphism. This follows from 1.2 because $\mathbf{R} f_{*} \mathcal{E}^{\bullet}(-r)$ is of finite homological dimension by Proposition 2.3 Finally, by Proposition 2.3. $\mathbf{R} \mathcal{H o m}_{\mathcal{O}_{z}}\left(\mathcal{E}^{\bullet}, f^{!} \mathcal{O}_{T}\right)$ is also of finite homological dimension over $T$.

Proposition 2.9. Assume that $X \rightarrow S$ is locally projective and let $\mathcal{K} \bullet$ be an object in $D_{c}^{b}\left(X \times{ }_{S} Y\right)$ of finite homological dimension over both $X$ and $Y$. The functor

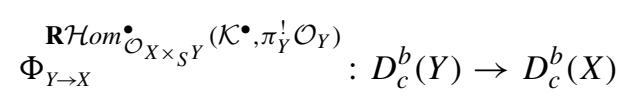

is a right adjoint to $\Phi_{X \rightarrow Y}^{\mathcal{K}}: D_{c}^{b}(X) \rightarrow D_{c}^{b}(Y)$. 
Proof. Since $\pi_{Y}$ is a locally projective morphism, Proposition 2.8 gives the result.

When the integral functor $\Phi_{X \rightarrow Y}^{\mathcal{K}^{\bullet}}$ is an equivalence between the bounded categories, Proposition 2.9 can be refined as follows:

Proposition 2.10. Let $X \rightarrow S$ and $Y \rightarrow S$ be projective morphisms and let $\mathcal{K} \bullet$ be a kernel in $D_{c}^{b}\left(X \times{ }_{S} Y\right)$ such that $\Phi_{X \rightarrow Y}^{\mathcal{K}}$ induces an equivalence

$$
\Phi_{X \rightarrow Y}^{\mathcal{K} \bullet}: D_{c}^{b}(X) \simeq D_{c}^{b}(Y)
$$

Then:

(1) $\mathcal{K} \bullet$ is of finite homological dimension over both $X$ and $Y$.

(2) The right adjoint to $\Phi_{X \rightarrow Y}^{\mathcal{K}^{\bullet}}: D_{c}^{b}(X) \simeq D_{c}^{b}(Y)$ is an integral functor, and its kernel $\mathbf{R} \mathcal{H o m}_{\mathcal{O}_{X \times{ }_{S}}}\left(\mathcal{K}^{\bullet}, \pi_{Y}^{!} \mathcal{O}_{Y}\right)$ is also of finite homological dimension over both $X$ and $Y$.

Proof. First, $\mathcal{K} \bullet$ is of finite homological dimension over $X$ by Proposition 2.7 We still do not know if $\mathcal{K} \bullet$ is of finite homological dimension over $Y$, so we cannot apply Proposition 2.9 However, we can proceed as follows: The functor $\Phi_{X^{\mathcal{K}}}^{\mathcal{K}^{\bullet}}: D(X) \rightarrow D(Y)$ over the whole derived category has a right adjoint $H$ (cf. formula $\sqrt{2.1}$ ). Since $\Phi_{X \rightarrow Y}^{\mathcal{K}^{\bullet}}: D_{c}^{b}(X) \simeq$ $D_{c}^{b}(Y)$ is an equivalence, it has a right adjoint $\bar{H}: D_{c}^{b}(Y) \rightarrow D_{c}^{b}(X)$. Let us prove that $H$ and $\bar{H}$ coincide over $D_{c}^{b}(X)$. For any $\mathcal{F}^{\bullet} \in D_{c}^{b}(X), \mathcal{G}^{\bullet} \in D_{c}^{b}(Y)$ one has

$$
\operatorname{Hom}_{D(X)}\left(\mathcal{F}^{\bullet}, \bar{H}\left(\mathcal{G}^{\bullet}\right)\right) \simeq \operatorname{Hom}_{D(Y)}\left(\Phi_{X \rightarrow Y}^{\mathcal{K}^{\bullet}}\left(\mathcal{F}^{\bullet}\right), \mathcal{G}^{\bullet}\right) \simeq \operatorname{Hom}_{D(X)}\left(\mathcal{F}^{\bullet}, H\left(\mathcal{G}^{\bullet}\right)\right)
$$

Hence, there is a morphism $\eta: \bar{H}\left(\mathcal{G}^{\bullet}\right) \rightarrow H\left(\mathcal{G}^{\bullet}\right)$ in $D^{+}(X)$ such that the induced morphism

$$
\operatorname{Hom}_{D(X)}^{i}\left(\mathcal{F}^{\bullet}, \bar{H}\left(\mathcal{G}^{\bullet}\right)\right) \rightarrow \operatorname{Hom}_{D(X)}^{i}\left(\mathcal{F}^{\bullet}, H\left(\mathcal{G}^{\bullet}\right)\right)
$$

is an isomorphism for any $\mathcal{F}^{\bullet} \in D_{c}^{b}(X)$ and every integer $i$. Let $\mathcal{C}^{\bullet} \in D^{+}(X)$ be the cone of $\eta$. Then $\operatorname{Hom}_{D(X)}^{i}\left(\mathcal{F}^{\bullet}, \mathcal{C}^{\bullet}\right)=0$ for any $\mathcal{F}^{\bullet} \in D_{c}^{b}(X)$ and any $i$. Taking $\mathcal{F}^{\bullet}=\mathcal{O}_{X}(r)$ for $r$ large enough, one concludes that $\mathcal{C}^{\bullet}=0$, so that $\bar{H} \simeq H$.

Now, for any $\mathcal{G}^{\bullet} \in D_{c}^{b}(Y)$ one finds that

$$
\bar{H}\left(\mathcal{G}^{\bullet} \otimes \mathcal{O}(r)\right) \simeq \mathbf{R} \pi_{X *}\left[\mathbf{R} \mathcal{H}_{o m_{\mathcal{O}{ }_{S} Y}}\left(\mathcal{K}^{\bullet}, \pi_{Y}^{!} \mathcal{G}^{\bullet}\right) \otimes \pi_{Y}^{*} \mathcal{O}(r)\right]
$$

is bounded for any integer $r$. By Lemma $2.5 \mathbf{R H o m}_{\mathcal{O}_{X \times{ }_{S} Y}}\left(\mathcal{K}^{\bullet}, \pi_{Y}^{!} \mathcal{G} \bullet\right)$ is bounded and so $\mathcal{K} \bullet$ has finite homological dimension over $Y$. It follows from Proposition 2.9 that $\bar{H}$ is an integral functor of kernel $\mathbf{R} \mathcal{H}_{\operatorname{om}_{\mathcal{O}_{X \times S}}}\left(\mathcal{K}^{\bullet}, \pi_{Y}^{!} \mathcal{O}_{Y}\right)$. Since $\bar{H}$ is an equivalence, its kernel is also of finite homological dimension over both $X$ and $Y$.

Proposition 2.11. Let $f: Z \rightarrow T$ be a locally projective morphism and $\mathcal{E} \bullet$ an object of $D_{c}^{b}(Z)$. If $\mathcal{E}^{\bullet}$ has finite homological dimension over $T$ then the natural morphism

$$
\mathcal{E}^{\bullet} \rightarrow \mathbf{R} \mathcal{H o m}_{\mathcal{O}_{Z}}\left(\mathbf{R} \mathcal{H o m}_{\mathcal{O}_{Z}}\left(\mathcal{E}^{\bullet}, f^{!} \mathcal{O}_{T}\right), f^{!} \mathcal{O}_{T}\right)
$$

is an isomorphism. 
Proof. The problem is local on $T$, so we can assume that $f$ is projective. Since $\mathbf{R} \mathcal{H}_{\text {om }_{\mathcal{O}_{Z}}}\left(\mathcal{E}^{\bullet}, f^{!} \mathcal{O}_{T}\right)$ is of finite homological dimension over $T$ by Proposition 2.8 the object $\mathbf{R} \mathcal{H o m}_{\mathcal{O}_{Z}}\left(\mathbf{R} \mathcal{H o m}_{\mathcal{O}_{Z}}\left(\mathcal{E}^{\bullet}, f^{!} \mathcal{O}_{T}\right), f^{!} \mathcal{O}_{T}\right)$ has bounded cohomology. Thus, by Lemma 2.5, to prove that

$$
\mathcal{E}^{\bullet} \rightarrow \mathbf{R} \mathcal{H o m}_{\mathcal{O}_{Z}}\left(\mathbf{R} \mathcal{H o m}_{\mathcal{O}_{Z}}\left(\mathcal{E}^{\bullet}, f^{!} \mathcal{O}_{T}\right), f^{!} \mathcal{O}_{T}\right)
$$

is an isomorphism we have to prove that the induced morphism

$$
\mathbf{R} f_{*}\left(\mathcal{E}^{\bullet}(r)\right) \rightarrow \mathbf{R} f_{*}\left(\mathbf{R} \mathcal{H o m}_{\mathcal{O}_{Z}}^{\bullet}\left(\mathbf{R H o m}_{\mathcal{O}_{Z}}\left(\mathcal{E}^{\bullet}, f^{!} \mathcal{O}_{T}\right), f^{!} \mathcal{O}_{T}\right)(r)\right)
$$

is an isomorphism for every integer $r$. Grothendieck duality implies that this morphism is the natural morphism $\mathbf{R} f_{*}\left(\mathcal{E}^{\bullet}(r)\right) \rightarrow\left(\mathbf{R} f_{*}\left(\mathcal{E}^{\bullet}(r)\right)\right)^{\vee \vee}$ (where for a complex $\mathcal{F}^{\bullet}$ we write $\mathcal{F}^{\bullet \vee}=\mathbf{R} \mathcal{H}^{\circ} m_{\mathcal{O}_{T}}\left(\mathcal{F}^{\bullet}, \mathcal{O}_{T}\right)$ for the derived dual), which is an isomorphism because $\mathbf{R} f_{*}(\mathcal{E} \bullet(r))$ is of finite homological dimension by Proposition 2.3 .

If $X$ is a proper scheme over a field, we denote by $\mathcal{E}^{\bullet \sharp}$ the "dual in the dualising complex", that is,

$$
\mathcal{E}^{\bullet \sharp}=\mathbf{R} \mathcal{H o m}_{\mathcal{O}_{X}}\left(\mathcal{E}^{\bullet}, \mathcal{D}_{X}^{\bullet}\right)
$$

If $X$ is projective, we can apply Proposition 2.11 to the projection $f: X \rightarrow$ Spec $k$. Since any bounded complex $\mathcal{E}^{\bullet}$ has finite homological dimension over Spec $k$ one has:

Corollary 2.12. Let $X$ be a projective scheme over a field. The contravariant functor $\sharp$ induces an anti-equivalence of categories $\sharp: D_{c}^{b}(X) \rightarrow D_{c}^{b}(X)$.

We finish this subsection with the following commutation property of integral functors and the functor $\sharp$ (cf. 2.5 ), which will be used in Theorems 3.6 and 4.4 .

Lemma 2.13. Let $X$ and $Y$ be projective schemes over a field and $\mathcal{K}^{\bullet} \in D_{c}^{b}(X \times Y) a$ kernel of finite homological dimension over $X$. Then

$$
\left(\Phi_{X \rightarrow Y}^{\mathcal{K} \bullet}\left(\mathcal{E}^{\bullet}\right)\right)^{\sharp} \simeq \underset{X \rightarrow Y}{\mathbf{R} \mathcal{H o m}_{\mathcal{O}_{X \times Y}}^{\bullet}}\left(\mathcal{K}^{\bullet}, \pi_{X}^{!} \mathcal{O}_{X}\right)\left(\mathcal{E}^{\bullet \sharp}\right) .
$$

for any object $\mathcal{E}^{\bullet}$ in $D_{c}^{b}(X)$.

Proof. On the one hand, we have

$$
\begin{aligned}
\left(\Phi_{X \rightarrow Y}^{\mathcal{K}^{\bullet}}\left(\mathcal{E}^{\bullet}\right)\right)^{\sharp} & \simeq \mathbf{R} \mathcal{H o m}_{\mathcal{O}_{Y}}\left(\mathbf{R} \pi_{Y *}\left(\pi_{X}^{*} \mathcal{E}^{\bullet} \stackrel{\mathbf{L}}{\otimes} \mathcal{K}^{\bullet}\right), \mathcal{D}_{Y}^{\bullet}\right) \\
& \simeq \mathbf{R} \pi_{Y *} \mathbf{R} \mathcal{H o m}_{\mathcal{O}_{X \times Y}}\left(\pi_{X}^{*} \mathcal{E} \bullet \stackrel{\mathbf{L}}{\otimes} \mathcal{K}^{\bullet}, \pi_{Y}^{!} \mathcal{D}_{Y}^{\bullet}\right) \\
& \simeq \mathbf{R} \pi_{Y *} \mathbf{R} \mathcal{H o m}_{\mathcal{O}_{X \times Y}}\left(\mathcal{K}^{\bullet}, \mathbf{R} \mathcal{H o m}_{\mathcal{O}_{X \times Y}}\left(\pi_{X}^{*} \mathcal{E}^{\bullet}, \pi_{Y}^{!} \mathcal{D}_{Y}^{\bullet}\right)\right)
\end{aligned}
$$

On the other hand,

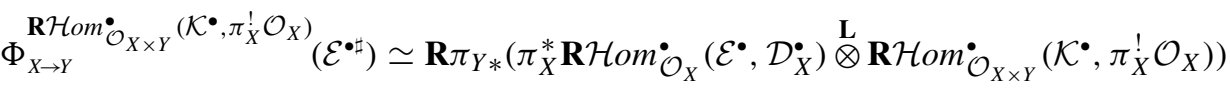

$$
\begin{aligned}
& \simeq \mathbf{R} \pi_{Y *} \mathbf{R} \mathcal{H o m}_{\mathcal{O}_{X \times Y}}\left(\mathcal{K} \cdot, \pi_{X}^{!} \mathbf{R} \mathcal{H o m}_{\mathcal{O}_{X}}\left(\mathcal{E}^{\bullet}, \mathcal{D}_{X}^{\bullet}\right)\right)
\end{aligned}
$$


where the last isomorphism is by Proposition 2.8. Hence, it is enough to see that $\mathbf{R} \mathcal{H o m}_{\mathcal{O}_{X \times Y}}\left(\pi_{X}^{*} \mathcal{E} \cdot \pi_{Y}^{!} \mathcal{D}_{Y}^{\bullet}\right) \simeq \pi_{X}^{!} \mathbf{R} \mathcal{H} \operatorname{Hom}_{\mathcal{O}_{X}}^{\bullet}\left(\mathcal{E}^{\bullet}, \mathcal{D}_{X}^{\bullet}\right)$. Since $\pi_{Y}^{!} \mathcal{D}_{Y}^{\bullet} \simeq \mathcal{D}_{X \times Y}^{\bullet} \simeq \pi_{X}^{!} \mathcal{D}_{X}^{\bullet}$, this follows from the isomorphisms

$$
\begin{aligned}
& \operatorname{Hom}_{D^{b}(X \times Y)}\left(\mathcal{F}^{\bullet}, \mathbf{R} \mathcal{H}_{\text {om }}^{\bullet} \mathcal{O}_{X \times Y}\left(\pi_{X}^{*} \mathcal{E}^{\bullet}, \mathcal{D}_{X \times Y}^{\bullet}\right)\right) \simeq \operatorname{Hom}_{D^{b}(X \times Y)}\left(\mathcal{F}^{\bullet} \otimes \pi_{X}^{*} \mathcal{E}^{\bullet}, \mathcal{D}_{X \times Y}^{\bullet}\right) \\
& \simeq \operatorname{Hom}_{D^{b}(X)}\left(\mathbf{R} \pi_{X *}\left(\mathcal{F}^{\bullet} \stackrel{\mathbf{L}}{\otimes} \pi_{X}^{*} \mathcal{E}^{\bullet}\right), \mathcal{D}_{X}^{\bullet}\right) \simeq \operatorname{Hom}_{D^{b}(X)}\left(\mathbf{R} \pi_{X *} \mathcal{F}^{\bullet} \stackrel{\mathbf{L}}{\left.\otimes \mathcal{E}^{\bullet}, \mathcal{D}_{X}^{\bullet}\right)}\right. \\
& \simeq \operatorname{Hom}\left(\mathbf{R} \pi_{X *} \mathcal{F}^{\bullet}, \mathbf{R} \mathcal{H o m}_{\mathcal{O}_{X}}\left(\mathcal{E}^{\bullet}, \mathcal{D}_{X}^{\bullet}\right)\right) \simeq \operatorname{Hom}_{D^{b}(X \times Y)}\left(\mathcal{F}^{\bullet}, \pi_{X}^{!} \mathbf{R} \mathcal{H o m}_{\mathcal{O}_{X}}\left(\mathcal{E}^{\bullet}, \mathcal{D}_{X}^{\bullet}\right)\right) \text {, }
\end{aligned}
$$

which hold for any object $\mathcal{F}^{\bullet}$ in $D_{c}^{b}(X \times Y)$.

\subsection{Restriction to fibres: a criterion for equivalence}

In this subsection we prove that, in a very general situation, to see that a relative integral functor is fully faithful (or an equivalence) it is enough to prove that its restriction to each fibre is fully faithful (or an equivalence). Using this result and the theorem of Seidel and Thomas that states that any twist functor along a spherical object is an equivalence of categories, we then construct a non-trivial auto-equivalence of the bounded derived category of an arbitrary genus one fibration.

The base field is here an algebraically closed field of arbitrary characteristic. Let $p: X \rightarrow S$ and $q: Y \rightarrow S$ be proper and flat morphisms. Let $\mathcal{K} \bullet$ be an object in $D_{c}^{b}\left(X \times_{S} Y\right)$ and $\Phi=\Phi_{X \rightarrow Y}^{\mathcal{K}^{\bullet}}$. For any closed point $s \in S$ we write $X_{s}=p^{-1}(s)$, $Y_{s}=q^{-1}(s)$, and denote by $\Phi_{s}: D^{-}\left(X_{s}\right) \rightarrow D^{-}\left(Y_{s}\right)$ the integral functor defined by $\mathcal{K}^{\bullet}{ }_{s}=\mathbf{L} j_{s}^{*} \mathcal{K} \bullet$, with $j_{s}: X_{s} \times Y_{S} \hookrightarrow X \times{ }_{S} Y$ the natural embedding.

When the kernel $\mathcal{K}^{\bullet} \in D_{c}^{b}\left(X \times_{S} Y\right)$ is of finite homological dimension over $X$, the functor $\Phi$ maps $D_{c}^{b}(X)$ into $D_{c}^{b}(Y)$, and $\mathcal{K}^{\bullet}{ }_{s} \in D_{c}^{b}\left(X_{s} \times Y_{s}\right)$ for any $s \in S$. Morever, since $q$ is flat, $\mathcal{K}^{\bullet}{ }_{s}$ is of finite homological dimension over $X_{s}$.

From the base-change formula (Proposition 1.1, see also [2]) we obtain

$$
\mathbf{L} j_{S}^{*} \Phi\left(\mathcal{F}^{\bullet}\right) \simeq \Phi_{s}\left(\mathbf{L} j_{s}^{*} \mathcal{F}^{\bullet}\right)
$$

for every $\mathcal{F}^{\bullet} \in D(X)$, where $j_{s}: X_{S} \hookrightarrow X$ and $j_{s}: Y_{S} \hookrightarrow Y$ are the natural embeddings. In this situation, the base-change formula also gives

$$
j_{S *} \Phi_{S}\left(\mathcal{G}^{\bullet}\right) \simeq \Phi\left(j_{S *} \mathcal{G}^{\bullet}\right)
$$

for every $\mathcal{G}^{\bullet} \in D\left(X_{S}\right)$.

Lemma 2.14. Let $\Psi: D^{-}(X) \rightarrow D^{-}(Y)$ be an integral functor whose kernel is an object of $D^{-}(X \times Y)$. For any integer $i$ there exists $r_{0}$ such that $\mathcal{H}^{i}\left(\Psi\left(\mathcal{G}^{\bullet}\right)\right) \simeq \mathcal{H}^{i}\left(\Psi\left(\sigma_{\geq r} \mathcal{G}^{\bullet}\right)\right)$ for every $r \leq r_{0}$ and any $\mathcal{G}^{\bullet} \in D^{-}(X)$, where $\sigma_{\geq r} \mathcal{G}^{\bullet}$ is the truncation (cf. [14, I§7]).

Proof. There exists an integer $m$ such that if $\mathcal{F}^{\bullet}$ is an object of $D^{-}(X)$ and $\mathcal{H}^{j}\left(\mathcal{F}^{\bullet}\right)=0$ for $j \geq s$, then $\mathcal{H}^{j}\left(\Psi\left(\mathcal{F}^{\bullet}\right)\right)=0$ for $j \geq s+m$. Let us take $r \leq i-1-m$ and let $\alpha_{r}: \mathcal{G}^{\bullet} \rightarrow \sigma_{\geq r}\left(\mathcal{G}^{\bullet}\right)$ be the natural morphism. Then $\mathcal{H}^{j}\left(\operatorname{Cone}\left(\alpha_{r}\right)\right)=0$ for $j \geq r$, so that $\mathcal{H}^{j}\left(\Psi\left(\right.\right.$ Cone $\left.\left.\left(\alpha_{r}\right)\right)\right)=0$ for $j \geq r+m$. In particular, $\mathcal{H}^{i-1}\left(\Psi\left(\operatorname{Cone}\left(\alpha_{r}\right)\right)\right)=$ 
$\mathcal{H}^{i}\left(\Phi^{-}\left(\right.\right.$Cone $\left.\left.\left(\alpha_{r}\right)\right)\right)=0$, and we finish by taking cohomology on the exact triangle

$$
\Psi\left(\mathcal{G}^{\bullet}\right) \rightarrow \Psi\left(\sigma_{\geq r}\left(\mathcal{G}^{\bullet}\right)\right) \rightarrow \Psi\left(\text { Cone }\left(\alpha_{r}\right)\right) \rightarrow \Psi\left(\mathcal{G}^{\bullet}\right)[1] .
$$

Proposition 2.15. Assume that $X \rightarrow S$ is locally projective and let $\mathcal{K} \cdot$ be an object in $D_{c}^{b}\left(X \times_{S} Y\right)$ of finite homological dimension over both $X$ and $Y$. The relative integral functor $\Phi=\Phi_{X \rightarrow Y}^{\mathcal{K}^{\bullet}}: D_{c}^{b}(X) \rightarrow D_{c}^{b}(Y)$ is fully faithful (resp. an equivalence) if and only if $\Phi_{s}: D_{c}^{b}\left(X_{S}\right) \rightarrow D_{c}^{b}\left(Y_{S}\right)$ is fully faithful (resp. an equivalence) for every closed point $s \in S$.

Proof. By Proposition 2.9, the integral functor $H=\Phi_{Y \rightarrow X}^{\mathbf{R} \mathcal{H}_{X \times Y}}{ }_{X \times Y}\left(\mathcal{K}^{\bullet}, \pi_{Y}^{!} \mathcal{O}_{Y}\right): D_{c}^{b}(X) \rightarrow$ $D_{c}^{b}(Y)$ is a right adjoint to $\Phi$. We can now proceed as in the proof of [16, Thm. 2.4], which we explain here in some more detail.

If $\Phi$ is fully faithful the unit morphism Id $\rightarrow H \circ \Phi$ is an isomorphism. Hence, given a closed point $s \in S$ and $\mathcal{G}^{\bullet} \in D_{c}^{b}\left(X_{s}\right)$, one has an isomorphism $j_{s *} \mathcal{G}^{\bullet} \rightarrow(H \circ \Phi)\left(j_{s *} \mathcal{G}^{\bullet}\right)$. Since $(H \circ \Phi)\left(j_{S *} \mathcal{G}^{\bullet}\right) \simeq j_{S *}\left(H_{S} \circ \Phi_{S}\right)\left(\mathcal{G}^{\bullet}\right)$ by 2.7) and $j_{s}$ is a closed immersion, the unit morphism $\mathcal{G}^{\bullet} \rightarrow\left(H_{S} \circ \Phi_{s}\right)\left(\mathcal{G}^{\bullet}\right)$ is an isomorphism; this proves that $\Phi_{s}$ is fully faithful.

Now assume that $\Phi_{s}$ is fully faithful for any closed point $s \in S$. To prove that $\Phi$ is fully faithful we have to see that the unit morphism $\eta$ : Id $\rightarrow H \circ \Phi$ is an isomorphism. For each $\mathcal{F}^{\bullet} \in D_{c}^{b}(X)$ we have an exact triangle

$$
\mathcal{F}^{\bullet} \stackrel{\eta\left(\mathcal{F}^{\bullet}\right)}{\longrightarrow}(H \circ \Phi)\left(\mathcal{F}^{\bullet}\right) \rightarrow \operatorname{Cone}\left(\eta\left(\mathcal{F}^{\bullet}\right)\right) \rightarrow \mathcal{F}^{\bullet}[1]
$$

Let us now fix a closed point $s \in S$. By [2.6, we have an exact triangle

$$
\mathbf{L} j_{s}^{*} \mathcal{F}^{\bullet} \rightarrow\left(H_{s} \circ \Phi_{s}\right)\left(\mathbf{L} j_{s}^{*} \mathcal{F}^{\bullet}\right) \rightarrow \mathbf{L} j_{s}^{*} \operatorname{Cone}\left(\eta\left(\mathcal{F}^{\bullet}\right)\right) \rightarrow \mathbf{L} j_{s}^{*} \mathcal{F}^{\bullet}[1]
$$

By Lemma 2.14, for every integer $i$ there exists $r$ small enough such that $\mathcal{H}^{i}\left(\mathbf{L} j_{s}^{*} \mathcal{F}^{\bullet}\right) \simeq$ $\mathcal{H}^{i}\left(\sigma_{\geq r} \mathbf{L} j_{s}^{*} \mathcal{F}^{\bullet}\right)$ and $\mathcal{H}^{i}\left(\left(H_{S} \circ \Phi_{s}\right)\left(\mathbf{L} j_{s}^{*} \mathcal{F}^{\bullet}\right)\right) \simeq \mathcal{H}^{i}\left(\left(H_{S} \circ \Phi_{s}\right)\left(\sigma_{\geq r} \mathbf{L} j_{s}^{*} \mathcal{F}^{\bullet}\right)\right)$. Since $\sigma_{\geq r} \mathbf{L} j_{s}^{*} \mathcal{F}^{\bullet}$ is a bounded complex, and $\eta_{s}:$ Id $\rightarrow H_{s} \circ \Phi_{s}$ is an isomorphism because $\Phi_{s}$ is fully faithful, one sees that $\mathcal{H}^{i}\left(\sigma_{\geq r} \mathbf{L} j_{s}^{*} \mathcal{F}^{\bullet}\right) \simeq \mathcal{H}^{i}\left[\left(H_{s} \circ \Phi_{s}\right)\left(\sigma_{\geq r} \mathbf{L} j_{s}^{*} \mathcal{F}^{\bullet}\right)\right]$. Thus $\mathbf{L} j_{s}^{*} \mathcal{F}^{\bullet} \rightarrow\left(H_{S} \circ \Phi_{s}\right)\left(\mathbf{L} j_{s}^{*} \mathcal{F}^{\bullet}\right)$ induces isomorphisms between all the cohomology sheaves, so that it is an isomorphism, and hence $\mathbf{L} j_{s}^{*} \operatorname{Cone}\left(\eta\left(\mathcal{F}^{\bullet}\right)\right)=0$. Since this holds for every closed point $s \in S$, we finish by [16, Lemma 2.3].

A similar argument gives the statement about equivalence.

Related results concerning Azumaya smooth varieties have been proved by Kuznetsov in [22, Prop. 2.44 and Thm. 2.46]. To apply his results to our situation, $X, Y$ and $S$ have to be smooth though the flatness conditions on $p$ and $q$ can be removed.

2.2.1. An auto-equivalence of the derived category of a genus one fibration. Let $p: X \rightarrow S$ be a genus one fibration, that is, a projective Gorenstein morphism whose fibres are curves with arithmetic genus $\operatorname{dim} H^{1}\left(X_{S}, \mathcal{O}_{X_{s}}\right)=1$ and have trivial dualising sheaf. No further assumptions on $S$ or $X$ are made here.

When the fibres are reduced of arithmetic genus one, the condition on the dualising sheaf is always satisfied. However, since nonreduced curves can also appear as degenerate 
fibres for such a genus one fibration, and for these curves the dualising sheaf need not be trivial (see for instance [12]), one has to assume it.

There are some cases where the structure of the singular fibres is known: For smooth elliptic surfaces over the complex numbers, the classification was given by Kodaira [20] and for smooth elliptic threefolds over a base field of characteristic different from 2 and 3 , they were classified by Miranda [23]. In both cases, the possible singular fibres are plane curves of the same type, the so-called Kodaira fibres. Nevertheless, in a genus one fibration non-plane curves could appear as degenerate fibres.

Since we are not putting any restriction on the characteristic of the base field or on the dimension of $X$, our genus one fibrations may have singular fibres other than the Kodaira fibres.

We consider the commutative diagram

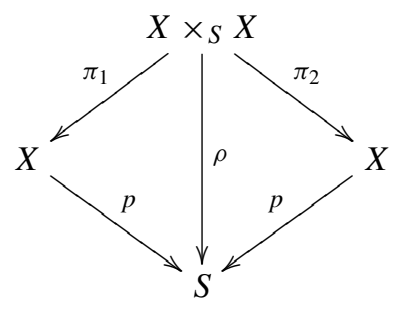

and the relative integral functor

$$
\Phi=\Phi_{X \rightarrow X}^{\mathcal{I}_{\Delta}}: D_{c}^{b}(X) \rightarrow D_{c}^{b}(X)
$$

with kernel the ideal sheaf $\mathcal{I}_{\Delta}$ of the relative diagonal immersion $\delta: X \hookrightarrow X \times{ }_{S} X$.

Proposition 2.16. The relative integral functor

$$
\Phi=\Phi_{X \rightarrow X}^{\mathcal{I}_{\Delta}}: D_{c}^{b}(X) \rightarrow D_{c}^{b}(X)
$$

defined by the ideal sheaf of the relative diagonal is an equivalence of categories.

Proof. To prove that $\mathcal{I}_{\Delta}$ is of finite homological dimension over both factors, it is enough to see that it is of finite homological dimension over the first factor because of its symmetry. By the exact sequence

$$
0 \rightarrow \mathcal{I}_{\Delta} \rightarrow \mathcal{O}_{X \times{ }_{S} X} \rightarrow \delta_{*} \mathcal{O}_{X} \rightarrow 0
$$

it suffices to see that $\delta_{*} \mathcal{O}_{X}$ has finite homological dimension over the first factor. We thus have to prove that for any $\mathcal{N}^{\bullet} \in D^{b}(X)$, the complex $\delta_{*} \mathcal{O}_{X} \otimes \pi_{1}^{*} \mathcal{N}^{\bullet}$ is bounded, and this follows from the projection formula for $\delta$.

For every closed point $s \in S$, the absolute functor $\Phi_{s}=\Phi_{X_{S} \rightarrow X_{S}}^{\mathcal{I}_{\Delta_{s}}}$ is equal to $T_{\mathcal{O}_{X_{S}}}[-1]$ where $T_{\mathcal{O}_{X_{S}}}$ denotes the twist functor along the object $\mathcal{O}_{X_{s}}$. Since $X_{s}$ is a genus one projective curve with trivial dualising sheaf, $\mathcal{O}_{X_{s}}$ is a spherical object. Thus, $T_{\mathcal{O}_{X_{s}}}$ is an equivalence of categories by [26] and we conclude by Proposition 2.15 
Similar results have been obtained by Burban and Kreussler in [11]. They proved a version of Proposition 2.16 in the case when the base field is of characteristic zero, $S$ and $X$ are reduced, $X$ is connected, the fibration $p: X \rightarrow S$ has only integral fibres and it has a section taking values in the smooth locus of $X$. None of these assumptions have been made in this section, though we assume for technical reasons that $S$ (and hence $X$ ) is separated.

Notice that, since we are working in the relative setting, the integral functor $\Phi_{X \rightarrow X}^{\mathcal{I}_{\Delta}}$ whose kernel is the ideal sheaf of the relative diagonal is not easily described as a twist functor. Actually, the twist functor $T_{\mathcal{O}_{X}}[-1]$ is the integral functor whose kernel is the ideal sheaf of the absolute diagonal immersion $X \hookrightarrow X \times X$. Even the latter functor may fail to be an equivalence because in general $\mathcal{O}_{X}$ is not spherical.

We shall give an alternative proof of Proposition 2.16, without using the work of Seidel and Thomas, in Subsection 3.4 .

\section{Full faithfulness criteria for integral functors}

\subsection{Strongly simple objects and spanning classes}

Recall that if $X$ is a Cohen-Macaulay scheme, for every point $x$ there exists a zero cycle $Z_{x}$ supported on $x$ defined locally by a regular sequence (cf. [16. Lemma 1.9]); we refer to such cycles as locally complete intersection or l.c.i. cycles. If $Z_{x} \hookrightarrow X$ is a l.c.i. cycle, by the Koszul complex theory, the structure sheaf $\mathcal{O}_{Z_{x}}$ has finite homological dimension as an $\mathcal{O}_{X}$-module.

In order to fix some notation, for any zero cycle $Z_{x}$ of $X$ and any scheme $S$, we shall denote by $j Z_{x}$ the immersion $Z_{x} \times S \hookrightarrow X \times S$.

For further use, we gather here two equivalent characterisations of objects of the derived category of an equidimensional scheme (that is, a scheme with all its irreducible components of the same dimension) defined by single sheaves supported on a closed subscheme. The statements are a slight generalisation of [16, Props. 1.15, 1.16] and the proofs are completely analogous.

Proposition 3.1. Let $X, Y$ be equidimensional Cohen-Macaulay schemes, $j: Y \hookrightarrow X a$ closed immersion of codimension $d$, and $\mathcal{K}^{\bullet}$ an object of $D_{c}^{b}(X)$. Assume that:

(1) If $x \in X-Y$ is a closed point, then $\mathbf{L}_{Z_{x}}^{*} \mathcal{K} \cdot=0$ for some l.c.i. zero cycle $Z_{x}$ supported on $x$.

(2) If $x \in Y$ is a closed point, then $L_{i} j_{Z_{x}}^{*} \mathcal{K}^{\bullet}=0$ for some l.c.i. zero cycle $Z_{x}$ supported on $x$ when either $i<0$ or $i>d$.

Then there is a sheaf $\mathcal{K}$ on $X$ whose topological support is contained in $Y$ and such that $\mathcal{K} \bullet \simeq \mathcal{K}$ in $D_{c}^{b}(X)$. Moreover, if $\mathcal{K} \bullet$ is non-zero, then the topological support of $\mathcal{K}$ is a union of irreducible components of $Y$. 
Proposition 3.2. Let $X, Y$ be equidimensional Cohen-Macaulay schemes of dimensions $m$ and $n$ respectively, $j: Y \hookrightarrow X$ a closed immersion, and $\mathcal{K} \bullet$ an object of $D_{c}^{b}(X)$. Assume that for any closed point $x \in X$ there is a l.c.i. zero cycle $Z_{x}$ supported on $x$ such that

$$
\operatorname{Hom}_{D(X)}^{i}\left(\mathcal{O}_{Z_{x}}, \mathcal{K}^{\bullet}\right)=0
$$

unless $x \in Y$ and $m \leq i \leq n$. Then there is a sheaf $\mathcal{K}$ on $X$ whose topological support is contained in $Y$ such that $\mathcal{K} \bullet \simeq \mathcal{K}$ in $D_{c}^{b}(X)$. Moreover, if $\mathcal{K}^{\bullet}$ is non-zero, then the topological support of $\mathcal{K}$ is a union of irreducible components of $Y$.

In this paper we only use the first part of the statement, namely the fact that $\mathcal{K} \bullet \simeq \mathcal{K}$ and its topological support is contained in $Y$.

Let $X$ and $Y$ be proper schemes. Assume that $X$ is Cohen-Macaulay. In this situation, the notion of strong simplicity is the following.

Definition 3.3. An object $\mathcal{K}^{\bullet}$ in $D_{c}^{b}(X \times Y)$ is strongly simple over $X$ if it satisfies the following conditions:

(1) For every closed point $x \in X$ there is a l.c.i. zero cycle $Z_{x}$ supported on $x$ such that

$$
\operatorname{Hom}_{D(Y)}^{i}\left(\Phi_{X \rightarrow Y}^{\mathcal{K} \bullet}\left(\mathcal{O}_{Z_{x_{1}}}\right), \Phi_{X \rightarrow Y}^{\mathcal{K} \bullet}\left(\mathcal{O}_{x_{2}}\right)\right)=0
$$

unless $x_{1}=x_{2}$ and $0 \leq i \leq \operatorname{dim} X$.

(2) $\operatorname{Hom}_{D(Y)}^{0}\left(\Phi_{X \rightarrow Y}^{\mathcal{K} \bullet}\left(\mathcal{O}_{x}\right), \Phi_{X \rightarrow Y}^{\mathcal{K}^{\bullet}}\left(\mathcal{O}_{x}\right)\right)=k$ for every closed point $x \in X$.

The last condition can be written as $\operatorname{Hom}_{D(Y)}^{0}\left(\mathbf{L} j_{x}^{*} \mathcal{K} \bullet, \mathbf{L} j_{x}^{*} \mathcal{K} \bullet\right)=k$, because the restriction $\mathbf{L} j_{x}^{*} \mathcal{K}^{\bullet}$ of $\mathcal{K}^{\bullet}$ to the fibre $j_{x}: Y \simeq\{x\} \times Y \hookrightarrow X \times Y$ can also be computed as $\Phi_{X \rightarrow Y}^{\mathcal{K}^{\bullet}}\left(\mathcal{O}_{x}\right)$. Then the objects $\mathbf{L} j_{x}^{*} \mathcal{K}^{\bullet}$ are simple and we may think of $\mathcal{K}^{\bullet}$ as a parametrisation of simple objects of $D_{c}^{b}(Y)$; this is why one calls $\mathcal{K}^{\bullet}$ a strongly simple object over $X$.

Remark 3.4. When $X$ and $Y$ are smooth, our definition is weaker than the usual one given by Bondal and Orlov (see [2]). Thus, Theorem 3.6 improves a result of Bondal and Orlov [6, Thm. 1.1]. Since both results characterise the full faithfulness of the corresponding integral functors, the two definitions turn out to be equivalent "a posteriori". In [16] we gave another notion of strongly simple objects for Gorenstein schemes. Again, Theorem 3.6 and the corresponding statement [16. Theorem 1.22] for the Gorenstein case prove that the two notions are equivalent in that situation.

3.1.1. Spanning classes. As in the Gorenstein case, the derived category $D_{c}^{b}(X)$ has a natural spanning class when $X$ is a Cohen-Macaulay scheme.

Lemma 3.5. If $X$ is a Cohen-Macaulay scheme, then the set

$$
\Omega=\left\{\mathcal{O}_{Z_{x}}: x \in X \text { a closed point, } Z_{x} \text { a l.c.i. zero cycle supported on } x\right\}
$$

is a spanning class for $D_{c}^{b}(X)$. 
Proof. Take a non-zero object $\mathcal{E}^{\bullet}$ in $D_{c}^{b}(X)$. On the one hand, for every 1.c.i. zero cycle as above there is a spectral sequence $E_{2}^{p, q}=\operatorname{Ext}_{\mathcal{O}_{X}}^{p}\left(\mathcal{H}^{-q}\left(\mathcal{E}^{\bullet}\right), \mathcal{O}_{Z_{x}}\right)$ converging to $E_{2}^{p+q}=$ $\operatorname{Hom}_{D(X)}^{p+q}\left(\mathcal{E}^{\bullet}, \mathcal{O}_{Z_{x}}\right)$. If $q_{0}$ is the maximum $q$ 's such that $\mathcal{H}^{q}\left(\mathcal{E}^{\bullet}\right) \neq 0$ and $x$ is a point of the support of $\mathcal{H}^{q}\left(\mathcal{E}^{\bullet}\right)$, then $E_{2}^{0,-q_{0}} \neq 0$ for every 1.c.i. zero cycle $Z_{x}$ and any non-zero element there survives to infinity. Thus $\operatorname{Hom}_{D(X)}^{-q_{0}}\left(\mathcal{E}^{\bullet}, \mathcal{O}_{Z_{x}}\right) \neq 0$.

On the other hand, by Proposition 3.2 with $Y=\emptyset$, if $\operatorname{Hom}_{D(X)}^{i}\left(\mathcal{O}_{Z_{x}}, \mathcal{E}^{\bullet}\right)=0$ for every $i$ and every $Z_{x}$, then $\mathcal{E}^{\bullet}=0$.

\subsection{A criterion in characteristic zero}

We now give a criterion for an integral functor between derived categories of CohenMacaulay proper schemes to be fully faithful.

Theorem 3.6. Let $X$ and $Y$ be proper schemes over an algebraically closed field of characteristic zero, and let $\mathcal{K} \bullet$ be an object in $D_{c}^{b}(X \times Y)$ of finite homological dimension over both $X$ and $Y$. Assume also that $X$ is projective, Cohen-Macaulay and integral. Then the functor $\Phi_{X \rightarrow Y}^{\mathcal{K}^{\bullet}}: D_{c}^{b}(X) \rightarrow D_{c}^{b}(Y)$ is fully faithful if and only if the kernel $\mathcal{K}^{\bullet}$ is strongly simple over $X$.

Proof. If the functor $\Phi_{X \rightarrow Y}^{\mathcal{K}^{\bullet}}$ is fully faithful, then $\mathcal{K}^{\bullet}$ is strongly simple over $X$.

Let us prove the converse. Before starting, we fix some notation: we denote by $\pi_{i}$ the projections of $X \times X$ onto its factors and $\Phi=\Phi_{X \rightarrow Y}^{\mathcal{K}^{\bullet}}$.

By Proposition 2.9. the integral functor $\left.H=\Phi_{Y \rightarrow X}^{\mathbf{R} \mathcal{H} o m_{X \times Y}^{\bullet}}{ }^{\bullet}, \mathcal{K}_{\dot{Y}}^{\bullet} \mathcal{O}_{Y}\right)$ is a right adjoint to $\Phi$. By [16, Prop. 1.18] it suffices to show that $H \circ \Phi$ is fully faithful. We know that the composition of integral functors is again an integral functor (cf. [16]), and so $H \circ \Phi \simeq$ $\Phi_{X \rightarrow X}^{\mathcal{M}^{\bullet}}$ with $\mathcal{M}^{\bullet} \in D_{c}^{b}(X \times X)$.

The strategy of the proof is similar to that of [16, Thm. 1.22]. We are going to prove that $\mathcal{M}^{\bullet} \simeq \delta_{*} \mathcal{N}$ where $\delta: X \hookrightarrow X \times X$ is the diagonal immersion and $\mathcal{N}$ is a line bundle

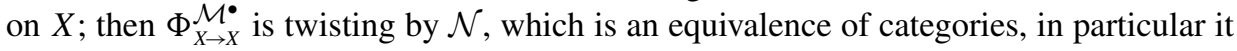
is fully faithful.

(a) $\mathbf{L} j_{x}^{*} \mathcal{M} \bullet$ is a single sheaf topologically supported on $x$. Thus $\mathcal{M}^{\bullet}$ is a single sheaf $\mathcal{M}$ topologically supported on the diagonal and flat over $X$ by the first projection by [7, Lemma 4.3].

Let us fix a closed point $\left(x_{1}, x_{2}\right) \in X \times X$ and consider the 1.c.i. zero cycle $Z_{x_{1}}$ of the first condition of the definition of strongly simple object. One has

$$
\operatorname{Hom}_{D(X)}^{i}\left(\mathcal{O}_{Z_{x_{1}}}, \Phi_{X \rightarrow X}^{\mathcal{M} \bullet}\left(\mathcal{O}_{x_{2}}\right)\right) \simeq \operatorname{Hom}_{D(Y)}^{i}\left(\Phi\left(\mathcal{O}_{Z_{x_{1}}}\right), \Phi\left(\mathcal{O}_{x_{2}}\right)\right)
$$

which is zero unless $x_{1}=x_{2}$ and $0 \leq i \leq m$ because $\mathcal{K}^{\bullet}$ is strongly simple. Applying Proposition 3.2 to the immersion $\left\{x_{2}\right\} \hookrightarrow X$ we see that $\Phi_{X \rightarrow X}^{\mathcal{M}} \mathcal{M}^{\bullet}\left(\mathcal{O}_{x_{2}}\right)$ reduces to a coherent sheaf topologically supported on $x_{2}$. Since $L j_{x}^{*} \mathcal{M} \simeq \Phi_{X \rightarrow X}^{\mathcal{M}}\left(\mathcal{O}_{x}\right)$, it follows that $\mathcal{M}^{\bullet}$ is a 
sheaf $\mathcal{M}$ whose support is contained in the diagonal and $\pi_{1 *} \mathcal{M}$ is locally free, where $\pi_{1}: X \times X \rightarrow X$ is the projection onto the first factor. The rank of $\pi_{1 *} \mathcal{M}$ cannot be zero, by condition (2) of strongly simple. Hence, the topological support of $\mathcal{M}^{\bullet}$ is exactly the diagonal.

(b) $\mathcal{M}$ is schematically supported on the diagonal, that is, $\mathcal{M}=\delta_{*} \mathcal{N}$ for a coherent sheaf $\mathcal{N}$ on $X$; moreover, $\mathcal{N}$ is a line bundle.

Let us denote by $\bar{\delta}: W \hookrightarrow X \times X$ the schematic support of $\mathcal{M}$ so that $\mathcal{M}=\bar{\delta}_{*} \mathcal{N}$ for a coherent sheaf $\mathcal{N}$ on $W$. Since $\mathcal{M}$ is topologically supported on the diagonal, the diagonal embedding $\delta$ factors through a closed immersion $\tau: X \hookrightarrow W$ which is a homeomorphism topologically.

Since $\mathcal{M}$ is flat over $X$ by $\pi_{1}, \mathcal{N}$ is flat over $X$ by the composition $\bar{\pi}_{1}=\pi_{1} \circ \bar{\delta}$ : $W \rightarrow X$. Moreover, $\bar{\pi}_{1}$ is a finite morphism, so that $\pi_{1 *} \mathcal{M} \simeq \bar{\pi}_{1 *} \mathcal{N}$ is locally free.

Now, as in the proof of [16, Thm. 1.22], to conclude it is enough to see that the rank of the locally free sheaf $\pi_{1 *} \mathcal{M}$ is one.

One has $\operatorname{Hom}^{0}\left(\mathcal{O}_{x}, \Phi_{X \rightarrow X}^{\mathcal{M}}\left(\mathcal{O}_{x}\right)\right) \simeq \operatorname{Hom}^{0}\left(\Phi\left(\mathcal{O}_{x}\right), \Phi\left(\mathcal{O}_{x}\right)\right) \simeq k$. Hence there is an injective morphism $\mathcal{O}_{x} \rightarrow \Phi_{X \rightarrow X}^{\mathcal{M}}\left(\mathcal{O}_{x}\right) \simeq j_{x}^{*} \mathcal{M}$. It suffices to show that it in isomorphism for at least one closed point $x$. If $C_{x}$ is the cokernel, we have to see that $\operatorname{Hom}^{0}\left(\mathcal{O}_{x}, C_{x}\right)=0$. Arguing as in [7] it is enough to show that $\operatorname{Hom}^{1}\left(\mathcal{O}_{x}, \mathcal{O}_{x}\right) \rightarrow$ $\operatorname{Hom}^{1}\left(\Phi_{X \rightarrow X}^{\mathcal{M}}\left(\mathcal{O}_{x}\right), \Phi_{X \rightarrow X}^{\mathcal{M}}\left(\mathcal{O}_{x}\right)\right)$ is injective.

Let us write $\widetilde{\mathcal{M}}=\mathbf{R} \mathcal{H o m}_{X \times X}^{\bullet}\left(\mathcal{M}, \pi_{1}^{!} \mathcal{O}_{X}\right)$, which is again a sheaf topologically supported on the diagonal and flat over $X$ by the first projection, because

$$
\pi_{1 *} \mathbf{R} \mathcal{H o m}_{X \times Y}^{\bullet}\left(\mathcal{M}, \pi_{1}^{!} \mathcal{O}_{X}\right) \simeq \mathbf{R} \mathcal{H o m}_{X \times Y}^{\bullet}\left(\pi_{1 *} \mathcal{M}, \mathcal{O}_{X}\right)
$$

and $\pi_{1 *} \mathcal{M}$ is locally free.

One has $\mathcal{O}_{x}^{\sharp} \simeq \mathcal{O}_{x}$ by relative duality for the closed immersion $\{x\} \hookrightarrow X$, so that $\mathbf{L} j_{x}^{*} \widetilde{\mathcal{M}} \simeq\left(\mathbf{L} j_{x}^{*} \mathcal{M}\right)^{\sharp}$ by Lemma 2.13 . Then

$$
\operatorname{Hom}^{0}\left(\mathbf{L} j_{x}^{*} \widetilde{\mathcal{M}}, \mathcal{O}_{x}\right) \simeq \operatorname{Hom}^{0}\left(\left(\mathbf{L} j_{x}^{*} \mathcal{M}\right)^{\sharp}, \mathcal{O}_{x}^{\sharp}\right) \simeq \operatorname{Hom}^{0}\left(\mathcal{O}_{x}, \mathbf{L} j_{x}^{*} \mathcal{M}\right) \simeq k
$$

because the functor $\sharp$ is an anti-equivalence of categories by Corollary 2.12 Hence, $\mathbf{L} j_{x}^{*} \widetilde{\mathcal{M}}$ is the sheaf of a zero cycle supported on $x$. We can then apply [7, Lemmas 5.2, 5.3] to $\widetilde{\mathcal{M}}$ to conclude that there exists a point $x$ such that

$$
\operatorname{Hom}^{1}\left(\mathcal{O}_{x}, \mathcal{O}_{x}\right) \rightarrow \operatorname{Hom}^{1}\left(\Phi_{X \rightarrow X}^{\widetilde{\mathcal{M}}}\left(\mathcal{O}_{x}\right), \Phi_{X \rightarrow X}^{\widetilde{\mathcal{M}}}\left(\mathcal{O}_{x}\right)\right) \simeq \operatorname{Hom}^{1}\left(\Phi_{X \rightarrow X}^{\mathcal{M}}\left(\mathcal{O}_{x}\right)^{\sharp}, \Phi_{X \rightarrow X}^{\mathcal{M}}\left(\mathcal{O}_{x}\right)^{\sharp}\right)
$$

is injective.

Now, again by Corollary 2.12 , we have a commutative diagram

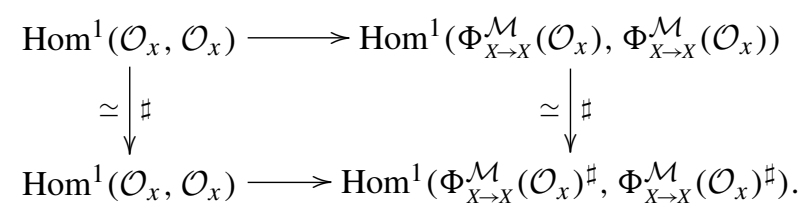

Since the bottom arrow is injective, the top arrow is injective as well and we conclude. 
The condition that the characteristic of the base field $k$ is zero is only used to prove that there exists a point $x$ such that the morphism (3.1) is injective [7, Lemmas 5.2, 5.3]. This is proved by showing that 3.1 is the Kodaira-Spencer map for the family $\widetilde{\mathcal{M}}$. Moreover, the sheaves $\widetilde{\mathcal{M}}_{x}$ define points of a Hilbert scheme and the above Kodaira-Spencer map is the composition of the tangent map to the map $x \mapsto \widetilde{\mathcal{M}}_{x}$, and the Kodaira-Spencer map for the universal family. The latter is an isomorphism and since $k$ has characteristic zero and $x \mapsto \widetilde{\mathcal{M}}_{x}$ is injective on closed points, its tangent map is injective at some point. It is this last statement which fails to be true in positive characteristic.

\subsection{A criterion in arbitrary characteristic}

As we showed in [16], Theorem 3.6 is no longer true in positive characteristic even in the smooth case. We reproduce here the counterexample given there. Let $X$ be a smooth projective scheme of dimension $m$ over a field $k$ of characteristic $p>0$, and $F: X \rightarrow X^{(p)}$ the relative Frobenius morphism [17, 3.1], which is topologically a homeomorphism. Let $\Gamma \hookrightarrow X \times X^{(p)}$ be the graph of $F$, whose associated integral functor is the direct image $F_{*}: D_{c}^{b}(X) \rightarrow D_{c}^{b}\left(X^{(p)}\right)$. Since $F_{*}\left(\mathcal{O}_{x}\right) \simeq \mathcal{O}_{F(x)}$, one easily sees that $\Gamma$ is strongly simple over $X$. However, $F_{*}\left(\mathcal{O}_{X}\right)$ is a locally free $\mathcal{O}_{X(p)}$-module of rank $p^{m}$ [17, 3.2], so that $\operatorname{Hom}_{D\left(X^{(p)}\right)}^{0}\left(F_{*}\left(\mathcal{O}_{X}\right), \mathcal{O}_{F(x)}\right) \simeq k^{p^{m}}$ whereas $\operatorname{Hom}_{D(X)}^{0}\left(\mathcal{O}_{X}, \mathcal{O}_{x}\right) \simeq k$; thus $F_{*}$ is not fully faithful.

Thus, in arbitrary characteristic we need another characterisation of those kernels which give rise to fully faithful integral functors. The right notion is the following.

Definition 3.7. An object $\mathcal{K} \bullet$ of $D_{c}^{b}(X \times Y)$ satisfies the Cohen-Macaulay orthonormality conditions over $X$ if it has the following properties:

(1) For every closed point $x \in X$ there is a l.c.i. zero cycle $Z_{x}$ supported on $x$ such that

$$
\operatorname{Hom}_{D(Y)}^{i}\left(\Phi_{X \rightarrow Y}^{\mathcal{K} \bullet}\left(\mathcal{O}_{Z_{x_{1}}}\right), \Phi_{X \rightarrow Y}^{\mathcal{K} \bullet}\left(\mathcal{O}_{x_{2}}\right)\right)=0
$$

unless $x_{1}=x_{2}$ and $0 \leq i \leq \operatorname{dim} X$.

(2) There exists a closed point $x$ such that at least one of the following conditions holds:

(2.1) $\operatorname{Hom}_{D(Y)}^{0}\left(\Phi_{X \rightarrow Y}^{\mathcal{K} \bullet}\left(\mathcal{O}_{X}\right), \Phi_{X \rightarrow Y}^{\mathcal{K} \bullet}\left(\mathcal{O}_{x}\right)\right) \simeq k$

(2.2) $\operatorname{Hom}_{D(Y)}^{0}\left(\Phi_{X \rightarrow Y}^{\mathcal{K} \bullet}\left(\mathcal{O}_{Z_{x}}\right), \Phi_{X \rightarrow Y}^{\mathcal{K}^{\bullet}}\left(\mathcal{O}_{x}\right)\right) \simeq k$ for any l.c.i. zero cycle $Z_{x}$ supported on $x$.

(2.3) $1 \leq \operatorname{dim}_{k} \operatorname{Hom}_{D(Y)}^{0}\left(\Phi_{X \rightarrow Y}^{\mathcal{K} \bullet}\left(\mathcal{O}_{Z_{x}}\right), \Phi_{X \rightarrow Y}^{\mathcal{K} \bullet}\left(\mathcal{O}_{Z_{x}}\right)\right) \leq l\left(\mathcal{O}_{Z_{x}}\right)$ for any l.c.i. zero cycle $Z_{x}$ supported on $x$, where $l\left(\mathcal{O}_{Z_{x}}\right)$ is the length of $\mathcal{O}_{Z_{x}}$.

Notice that now the objects $\mathbf{L} j_{x}^{*} \mathcal{K} \bullet$ are not required to be simple; hence we avoid the word simple in the denomination of the objects satisfying the above conditions and return to something closer to Bondal and Orlov's original way of describing them.

We now prove a variant of Theorem 3.6 which is valid in arbitrary characteristic. Here, the requirement that $X$ is integral can be relaxed. 
Theorem 3.8. Let $X$ and $Y$ be proper schemes over an algebraically closed field of arbitrary characteristic, and let $\mathcal{K}^{\bullet}$ be an object in $D_{c}^{b}(X \times Y)$ of finite homological dimension over both $X$ and $Y$. Assume also that $X$ is projective, Cohen-Macaulay, equidimensional and connected. Then the functor $\Phi_{X \rightarrow Y}^{\mathcal{K}^{\bullet}}: D_{c}^{b}(X) \rightarrow D_{c}^{b}(Y)$ is fully faithful if and only if the kernel $\mathcal{K} \bullet$ satisfies the Cohen-Macaulay orthonormality conditions over $X$ (Definition 3.7.

Proof. The direct implication is immediate. For the converse we proceed as in the proof of 3.6. As there, using condition (1) of Definition 3.7, one sees that $\Phi$ has a right adjoint $H$ and that $H \circ \Phi \simeq \Phi_{X \rightarrow X}^{\mathcal{M}}$, where $\mathcal{M}$ is a sheaf whose support is contained in the diagonal and $\pi_{1 *} \mathcal{M}$ is locally free. Since $X$ is connected, we can consider the rank $r$ of $\pi_{1 *} \mathcal{M}$, which is non-zero by condition (2) of Definition 3.7 thus the support of $\mathcal{M}$ is the diagonal. To conclude, we only have to prove that $r=1$.

If $\mathcal{K} \bullet$ satisfies $(2.1)$ of Definition 3.7, then

$$
\operatorname{Hom}_{D(X)}^{0}\left(\mathcal{O}_{X}, \Phi_{X \rightarrow X}^{\mathcal{M}}\left(\mathcal{O}_{x}\right)\right) \simeq \operatorname{Hom}_{D(Y)}^{0}\left(\Phi_{X \rightarrow Y}^{\mathcal{K}^{\bullet}}\left(\mathcal{O}_{X}\right), \Phi_{X \rightarrow Y}^{\mathcal{K}^{\bullet}}\left(\mathcal{O}_{x}\right)\right) \simeq k
$$

Hence $\Phi_{X \rightarrow X}^{\mathcal{M}}\left(\mathcal{O}_{x}\right) \simeq \mathcal{O}_{x}$ and $r=1$.

If $\mathcal{K} \cdot$ satisfies (2.2) of Definition 3.7, then

$$
\operatorname{Hom}_{D(X)}^{0}\left(\mathcal{O}_{Z_{x}}, \Phi_{X \rightarrow X}^{\mathcal{M}}\left(\mathcal{O}_{x}\right)\right) \simeq \operatorname{Hom}_{D(Y)}^{0}\left(\Phi_{X \rightarrow Y}^{\mathcal{K} \bullet}\left(\mathcal{O}_{Z_{x}}\right), \Phi_{X \rightarrow Y}^{\mathcal{K} \bullet}\left(\mathcal{O}_{x}\right)\right) \simeq k
$$

for any l.c.i. zero cycle $Z_{x}$. Hence $\Phi_{X \rightarrow X}^{\mathcal{M}}\left(\mathcal{O}_{x}\right) \simeq \mathcal{O}_{x}$ and $r=1$.

Finally, assume that $\mathcal{K}^{\bullet}$ satisfies (2.3) of Definition 3.7, and let us prove that then condition (2.2) of Definition 3.7 holds as well.

If $\widetilde{\mathcal{M}}^{\bullet}=\mathbf{R} \mathcal{H o m}_{\mathcal{O}_{X \times X}}^{\bullet}\left(\mathcal{M}, \pi_{1}^{!} \mathcal{O}_{X}\right)$, then proceeding as in the proof of Theorem 3.6 , one finds that $\widetilde{\mathcal{M}} \cdot$ is a sheaf $\widetilde{\mathcal{M}}$ supported topologically on the diagonal, and so $\pi_{1 *} \widetilde{\mathcal{M}}$ is locally free. It follows that the functor $\Phi_{X \rightarrow X}^{\mathcal{M}}$ has a left adjoint $G$, defined as $G\left(\mathcal{F}^{\bullet}\right)=\mathbf{R} \pi_{1 *}\left(\pi_{2}^{*} \mathcal{F} \bullet \stackrel{\mathbf{L}}{\otimes} \widetilde{\mathcal{M}}\right)$. This can be seen as follows: one knows

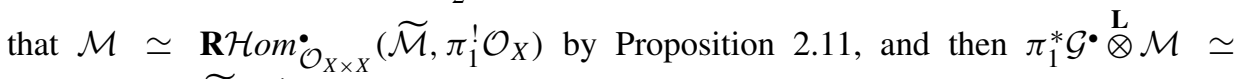
$\mathbf{R} \mathcal{H}^{\circ} \dot{\mathcal{O}}_{X \times X}\left(\widetilde{\mathcal{M}}, \pi_{1}^{!} \mathcal{G}^{\bullet}\right)$ by Proposition 2.8 ; thus

$$
\operatorname{Hom}\left(\mathcal{F}^{\bullet}, \Phi_{X \rightarrow X}^{\mathcal{M}}\left(\mathcal{G}^{\bullet}\right)\right) \simeq \operatorname{Hom}\left(\pi_{2}^{*} \mathcal{F}^{\bullet}, \mathbf{R} \mathcal{H}_{\operatorname{om}^{\bullet}} \mathcal{O}_{X \times X}\left(\widetilde{\mathcal{M}}, \pi_{1}^{!} \mathcal{G}^{\bullet}\right)\right) \simeq \operatorname{Hom}\left(G\left(\mathcal{F}^{\bullet}\right), \mathcal{G}^{\bullet}\right)
$$

which proves that $G$ is a left adjoint to $\Phi_{X \rightarrow X}^{\mathcal{M}}$. Thus, condition (2.2) is equivalent to $\operatorname{Hom}_{D(X)}^{0}\left(G\left(\mathcal{O}_{Z_{x}}\right), \mathcal{O}_{x}\right) \simeq k$.

We know that $\mathcal{M}$ is a sheaf topologically supported on the diagonal and $\pi_{1 *} \mathcal{M}$ is locally free. Therefore, if $\mathcal{F}$ is a sheaf, $\Phi_{X \rightarrow X}^{\mathcal{M}}(\mathcal{F})$ is also a sheaf and the functor $\mathcal{F} \mapsto$ $\phi(\mathcal{F})=\Phi_{X \rightarrow X}^{\mathcal{M}}(\mathcal{F})$ is exact. One has $\mathcal{H}^{i}(G(\mathcal{F}))=0$ for $i>0$ because $\widetilde{\mathcal{M}}$ is topologically supported on the diagonal. Hence, $\operatorname{Hom}_{D(X)}\left(G\left(\mathcal{F}_{1}\right), \mathcal{F}_{2}\right) \simeq \operatorname{Hom}_{D(X)}\left(G^{0}\left(\mathcal{F}_{1}\right), \mathcal{F}_{2}\right)$ for any sheaves $\mathcal{F}_{1}, \mathcal{F}_{2}$, where $G^{0}=\mathcal{H}^{0} \circ G$. This has two consequences: first, $G^{0}$ is a left adjoint to $\phi$; second, there are isomorphisms

$$
\operatorname{Hom}_{D(X)}^{0}\left(G\left(\mathcal{O}_{Z_{x}}\right), \mathcal{O}_{x}\right) \simeq \operatorname{Hom}_{D(X)}^{0}\left(G^{0}\left(\mathcal{O}_{Z_{x}}\right), \mathcal{O}_{x}\right) \simeq \operatorname{Hom}_{\mathcal{O}_{Z_{x}}}\left(j_{Z_{x}}^{*} G^{0}\left(\mathcal{O}_{Z_{x}}\right), \mathcal{O}_{x}\right)
$$


so that we are reduced to proving that $\operatorname{Hom}_{\mathcal{O}_{Z_{x}}}\left(j_{Z_{x}}^{*} G^{0}\left(\mathcal{O}_{Z_{x}}\right), \mathcal{O}_{x}\right) \simeq k$. Clearly, it is enough to see that $j_{Z_{x}}^{*} G^{0}\left(\mathcal{O}_{Z_{x}}\right) \simeq \mathcal{O}_{Z_{x}}$.

Using the exactness of $\phi$, one proves by induction on the length $\ell(\mathcal{F})$ that the unit map $\mathcal{F} \rightarrow \phi(\mathcal{F})$ is injective for any sheaf $\mathcal{F}$ supported on $x$. It then follows that the morphism $G^{0}(\mathcal{F}) \rightarrow \mathcal{F}$ is an epimorphism. To see that this is indeed the case, we only have to prove that for every sheaf $\mathcal{F}^{\prime}$ supported on the point $x$, the morphism $\operatorname{Hom}\left(\mathcal{F}, \mathcal{F}^{\prime}\right) \rightarrow$ $\operatorname{Hom}\left(G^{0}(\mathcal{F}), \mathcal{F}^{\prime}\right)$ is injective. By the adjunction formula, this morphism identifies with the morphism $\operatorname{Hom}\left(\mathcal{F}, \mathcal{F}^{\prime}\right) \rightarrow \operatorname{Hom}\left(\mathcal{F}, \phi\left(\mathcal{F}^{\prime}\right)\right)$, which is injective because $\mathcal{F}^{\prime} \rightarrow \phi\left(\mathcal{F}^{\prime}\right)$ is.

In particular, $\eta: G^{0}\left(\mathcal{O}_{Z_{x}}\right) \rightarrow \mathcal{O}_{Z_{x}}$ is surjective, and $\operatorname{dim} \operatorname{Hom}_{D(X)}^{0}\left(G^{0}\left(\mathcal{O}_{Z_{x}}\right), \mathcal{O}_{Z_{x}}\right) \geq$ $\ell\left(\mathcal{O}_{Z_{x}}\right)$. Thus, by condition (2.3) of Definition 3.7. $\operatorname{dim} \operatorname{Hom}_{D(X)}^{0}\left(G^{0}\left(\mathcal{O}_{Z_{x}}\right), \mathcal{O}_{Z_{x}}\right)=$ $\ell\left(\mathcal{O}_{Z_{x}}\right)$. Since $\mathcal{O}_{Z_{x}}$ is free, the exact sequence of $\mathcal{O}_{Z_{x}}$-modules

$$
0 \rightarrow \mathcal{N} \rightarrow j_{Z_{x}}^{*} G^{0}\left(\mathcal{O}_{Z_{x}}\right) \stackrel{j_{Z_{x}}^{*}(\eta)}{\longrightarrow} \mathcal{O}_{Z_{x}} \rightarrow 0
$$

splits, so that

$0 \rightarrow \operatorname{Hom}_{\mathcal{O}_{Z_{x}}}\left(\mathcal{O}_{Z_{x}}, \mathcal{O}_{Z_{x}}\right) \rightarrow \operatorname{Hom}_{\mathcal{O}_{Z_{x}}}\left(j_{Z_{x}}^{*} G^{0}\left(\mathcal{O}_{Z_{x}}\right), \mathcal{O}_{Z_{x}}\right) \rightarrow \operatorname{Hom}_{\mathcal{O}_{Z_{x}}}\left(\mathcal{N}, \mathcal{O}_{Z_{x}}\right) \rightarrow 0$

is an exact sequence. Moreover, $\operatorname{Hom}_{\mathcal{O}_{Z_{x}}}\left(\mathcal{N}, \mathcal{O}_{Z_{x}}\right)=0$ because the first two terms have the same dimension. Let us see that this implies $\mathcal{N}=0$. If $\mathcal{O}_{x} \rightarrow \mathcal{O}_{Z_{x}}$ is a non-zero, and hence injective, morphism, we have $\operatorname{Hom}_{\mathcal{O}_{z_{x}}}\left(\mathcal{N}, \mathcal{O}_{x}\right)=0$ so that $\mathcal{N}=0$ by Nakayama's lemma.

Remark 3.9. Even if the base field has characteristic zero, there is no obvious direct relationship between strong simplicity and the Cohen-Macaulay orthonormality conditions for a kernel $\mathcal{K}^{\bullet}$ in $D_{c}^{b}(X \times Y)$, despite the fact that they are equivalent due to Theorems 3.6 and 3.8 . Thus, Theorem 3.8 is actually a new characterisation of which kernels induce a fully faithful integral functor.

If we wish to directly compare strong simplicity with the Cohen-Macaulay orthonormality conditions, we see that the first condition in both definitions is the same; however, the second condition of strong simplicity is a property that has to be satisfied at every closed point for one cycle supported on the point, whereas two of the forms of the second condition of the Cohen-Macaulay orthonormality conditions refer to a property which has to be satisfied for all zero cycles supported on only one closed point.

\subsection{A criterion in the relative setting}

In the relative situation the notions of strongly simple object and of an object satisfying the Cohen-Macaulay orthonormality conditions are the following.

Definition 3.10. Assume that $X \rightarrow S$ is Cohen-Macaulay. An object $\mathcal{K}^{\bullet} \in D_{c}^{b}\left(X \times{ }_{S} Y\right)$ is relatively strongly simple (resp. satisfies the relative Cohen-Macaulay orthonormality conditions) over $X$ if $\mathcal{K}^{\bullet}{ }_{s}$ is strongly simple (resp. satisfies the Cohen-Macaulay orthonormality conditions) over $X_{s}$ for every closed point $s \in S$. 
As a corollary of Proposition 2.15 and Theorems 3.6 and 3.8 , we obtain the following result.

Theorem 3.11. Let $X \rightarrow S$ and $Y \rightarrow S$ be proper and flat morphisms. Assume also that $X \rightarrow S$ is locally projective with Cohen-Macaulay, equidimensional, and connected fibres. Let $\mathcal{K} \bullet$ be an object in $D_{c}^{b}\left(X \times_{S} Y\right)$ of finite homological dimension over both $X$ and $Y$.

(1) Assume that we are in characteristic 0 and $X \rightarrow S$ has integral fibres. The relative integral functor $\Phi_{X \rightarrow Y}^{\mathcal{K}}: D_{c}^{b}(X) \rightarrow D_{c}^{b}(Y)$ is fully faithful if and only if $\mathcal{K} \bullet$ is relatively strongly simple over $X$.

(2) In arbitrary characteristic, $\Phi_{X \rightarrow Y}^{\mathcal{K}^{\bullet}}: D_{c}^{b}(X) \rightarrow D_{c}^{b}(Y)$ is fully faithful if and only if $\mathcal{K}^{\bullet}$ satisfies the relative Cohen-Macaulay orthonormality conditions over $X$.

3.4.1. Application to genus one fibrations in arbitrary characteristic. We now apply Theorem 3.11 to give an alternative proof for Proposition 2.16 without using spherical objects.

With the same notation as in Subsection 2.2.1 and taking into account the symmetry of $\mathcal{I}_{\Delta}$, to conclude that the functor $\Phi=\Phi_{X \rightarrow X}^{\mathcal{I}_{\Delta}}$ is an auto-equivalence of $D_{c}^{b}(X)$, it is enough to prove that $\mathcal{I}_{\Delta}$ satisfies the relative Cohen-Macaulay orthonormality conditions over the first factor. We fix a closed point $s \in S$ and consider two points $x$ and $\bar{x}$ in the fibre $X_{s}$.

Let $Z_{x} \hookrightarrow X_{s}$ be a 1.c.i. zero cycle supported on $x$ of length $\ell$ defined by an ideal $\mathcal{I}_{Z_{x}}$; then $\mathcal{I}_{Z_{x}}$ is an invertible sheaf of $\mathcal{O}_{X_{s}}$-modules. We denote by $\pi: Z_{x} \times X_{S} \rightarrow X_{S}$ the second projection and by $\mathcal{J}_{Z_{x}}$ the push-forward by $\pi$ of the ideal of the graph of $Z_{x} \hookrightarrow X_{s}$. We have a commutative diagram with exact rows

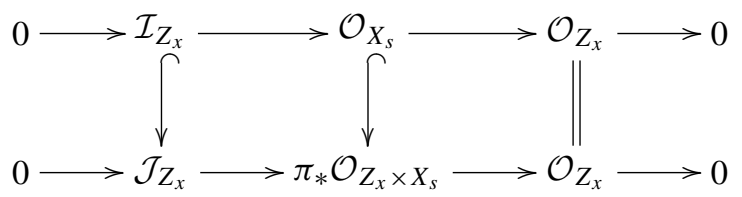

Since $\pi_{*} \mathcal{O}_{Z_{x} \times X_{s}}$ is a free $\mathcal{O}_{X_{s}}$-module of rank $\ell$, and $\pi$ has a section, one easily sees that the quotient sheaf $\pi_{*} \mathcal{O}_{Z_{x} \times X_{s}} / \mathcal{O}_{X_{s}}$ is free of rank $\ell-1$ and we have an exact sequence

$$
0 \rightarrow \mathcal{I}_{Z_{x}} \rightarrow \mathcal{J}_{Z_{x}} \rightarrow \mathcal{O}_{X_{s}}^{\oplus(\ell-1)} \rightarrow 0
$$

which proves that $\mathcal{J}_{Z_{x}}$ is locally free of rank $\ell$.

One has $\Phi_{s}\left(\mathcal{O}_{Z_{x}}\right) \simeq \mathcal{J}_{Z_{x}}$ and $\Phi_{s}\left(\mathcal{O}_{x}\right) \simeq \mathcal{I}_{x}$, where $\mathcal{I}_{x}$ is the ideal of the point $x$; we then have to compute the groups $\operatorname{Hom}_{D\left(X_{s}\right)}^{i}\left(\mathcal{J}_{Z_{x}}, \mathcal{I}_{\bar{x}}\right)$. Since $\mathcal{J}_{Z_{x}}$ is locally free, we know that $\operatorname{Hom}_{D\left(X_{s}\right)}^{i}\left(\mathcal{J}_{Z_{x}}, \mathcal{I}_{\bar{x}}\right)=0$ unless $i=0,1$, so we have to worry only about the cases $i=0$ and $i=1$.

We have

$$
\operatorname{Hom}^{0}\left(\pi_{*} \mathcal{O}_{Z_{x} \times X_{s}}, \mathcal{I}_{\bar{x}}\right) \simeq \operatorname{Hom}^{0}\left(\mathcal{O}_{X_{s}}^{\oplus \ell}, \mathcal{I}_{\bar{x}}\right) \simeq H^{0}\left(X_{s}, \mathcal{I}_{\bar{x}}\right)^{\oplus \ell}=0
$$


Then, from the bottom row of diagram (3.2) we obtain the following diagram:

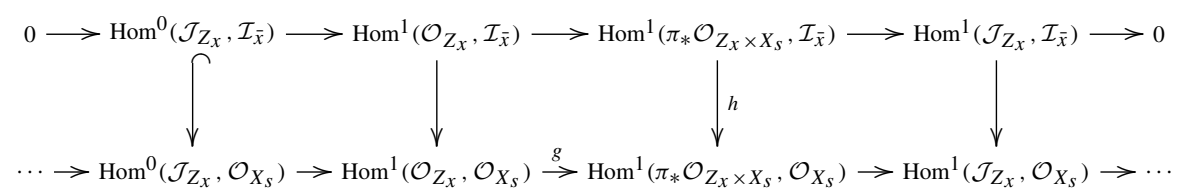

(a) The morphisms $h$ and $g$ are isomorphisms.

The fact that $h$ is an isomorphism follows from the formula $\pi_{*} \mathcal{O}_{Z_{x} \times X_{s}} \simeq \mathcal{O}_{X_{s}}^{\oplus \ell}$. To prove that $g$ is also an isomorphism, we first notice that $g$ is the morphism obtained by applying the functor $\operatorname{Hom}^{1}\left(-, \mathcal{O}_{X_{s}}\right)$ to the projection $\varpi: \pi_{*} \mathcal{O}_{Z_{x} \times X_{s}} \rightarrow \mathcal{O}_{Z_{x}}$. By duality, $\operatorname{Hom}^{1}\left(-, \mathcal{O}_{X_{s}}\right) \simeq H^{0}\left(X_{s},-\right)^{*}$ and one finishes because $\varpi: \pi_{*} \mathcal{O}_{Z_{x} \times X_{s}} \rightarrow \mathcal{O}_{Z_{x}}$ induces an isomorphism between the corresponding spaces of global sections.

(b) Condition (1) of Definition 3.7 holds.

It suffices to see that $\operatorname{Hom}^{1}\left(\mathcal{O}_{Z_{x}}, \mathcal{I}_{\bar{x}}\right) \rightarrow \operatorname{Hom}^{1}\left(\pi_{*} \mathcal{O}_{Z_{x} \times X_{s}}, \mathcal{I}_{\bar{x}}\right)$ is an isomorphism when $x \neq \bar{x}$. In this case, the second vertical arrow of diagram (3.3) is an isomorphism, so that the above morphism is identified with $g$, which is an isomorphism.

(c) Condition (2.2) of Definition 3.7 holds.

We have to prove that $\operatorname{Hom}^{0}\left(\mathcal{J}_{Z_{x}}, \mathcal{I}_{x}\right) \simeq k$. From diagram $(3.3), \operatorname{Hom}^{0}\left(\mathcal{J}_{Z_{x}}, \mathcal{I}_{x}\right)$ is isomorphic to the kernel of the second vertical arrow $\operatorname{Hom}^{1}\left(\mathcal{O}_{Z_{x}}, \mathcal{I}_{x}\right) \rightarrow \operatorname{Hom}^{1}\left(\mathcal{O}_{Z_{x}}, \mathcal{O}_{X_{s}}\right)$, which is isomorphic to $\operatorname{Hom}^{0}\left(\mathcal{O}_{Z_{x}}, \mathcal{O}_{x}\right) \simeq k$.

Notice that the proof of this result does not use spanning classes and is valid in any characteristic.

\section{Fourier-Mukai partners}

When two proper schemes $X$ and $Y$ have equivalent derived categories $D_{c}^{b}(X) \simeq D_{c}^{b}(Y)$, they are called $D$-equivalent. When the equivalence is given by an integral functor (such functors are called Fourier-Mukai functors), we have the following more restrictive notion.

Definition 4.1. Two proper schemes $X$ and $Y$ are Fourier-Mukai partners if there is a Fourier-Mukai functor

$$
\Phi_{X \rightarrow Y}^{\mathcal{K} \bullet}: D_{c}^{b}(X) \simeq D_{c}^{b}(Y)
$$

Due to Orlov's representation theorem [25], if $X$ and $Y$ are smooth and projective then they are $D$-equivalent if and only if they are Fourier-Mukai partners. Since the validity of Orlov's theorem for singular varieties is still unknown, we shall adopt the (hopefully provisional) notion of Fourier-Mukai partners.

In the rest of the section, $X$ and $Y$ are projective and $\mathcal{K}^{\bullet}$ is a kernel in $D_{c}^{b}(X \times Y)$ such that $\Phi_{X \rightarrow Y}^{\mathcal{K}^{\bullet}}$ is an equivalence $D_{c}^{b}(X) \simeq D_{c}^{b}(Y)$. 
Proposition 4.2. There is a natural isomorphism

$$
\mathbf{R} \mathcal{H o m}_{\mathcal{O}_{X \times Y}}\left(\mathcal{K} \cdot, \pi_{X}^{!} \mathcal{O}_{X}\right) \simeq \mathbf{R} \mathcal{H o m}_{\mathcal{O}_{X \times Y}}\left(\mathcal{K} \cdot, \pi_{Y}^{!} \mathcal{O}_{Y}\right) .
$$

Proof. By Proposition 2.10 the right adjoint to $\Phi_{X \rightarrow Y}^{\mathcal{K}}$ is $\left.\Phi_{Y \rightarrow X}^{\mathbf{R} \mathcal{H}^{\bullet}} \dot{\mathcal{O}}_{X \times Y}{ }^{\bullet}, \mathcal{K}_{Y}^{\bullet} \mathcal{O}_{Y}\right)$ and the right adjoint to the latter functor is $\Phi_{X \rightarrow Y}^{\mathcal{L}_{X}^{\bullet}}$ where

$$
\mathcal{L}^{\bullet}=\mathbf{R H o m}_{\mathcal{O}_{X \times Y}}^{\bullet}\left(\mathbf{R} \mathcal{H o m}_{\mathcal{O}_{X \times Y}}^{\bullet}\left(\mathcal{K}^{\bullet}, \pi_{Y}^{!} \mathcal{O}_{Y}\right), \pi_{X}^{!} \mathcal{O}_{X}\right) .
$$

Since the left and right adjoint of an equivalence are naturally isomorphic, one finds that $\Phi_{X \rightarrow Y}^{\mathcal{K}^{\bullet}} \simeq \Phi_{X \rightarrow Y}^{\mathcal{L}^{\bullet}}$.

Moreover, for any scheme $T$ we can consider $X_{T}=X \times T$ and $Y_{T}=Y \times T$. The kernel $\mathcal{K}^{\bullet}$ gives rise to a relative kernel $\mathcal{K}^{\bullet} T_{T}=\pi_{X \times Y}^{*} \mathcal{K} \bullet \in D_{c}^{b}\left(X_{T} \times_{T} Y_{T}\right)$, where $\pi_{X \times Y}$ is the projection $X_{T} \times_{T} Y_{T} \simeq X \times Y \times T \rightarrow X \times Y$. This relative kernel is of finite homological dimension over both $X_{T}$ and $Y_{T}$, and the relative integral functor $\Phi_{X_{T} \rightarrow Y_{T}}^{\mathcal{K}^{\bullet}{ }_{T}}: D_{c}^{b}\left(X_{T}\right) \rightarrow D_{c}^{b}\left(Y_{T}\right)$ is an equivalence by Proposition 2.15 Arguing as above, we get $\Phi_{X_{T} \rightarrow Y_{T}}^{\mathcal{K}_{T}} \simeq \Phi_{X_{T} \rightarrow Y_{T}}^{\mathcal{L}_{T}^{\bullet}}$. If we take $T=X$ and apply the above isomorphism to the sheaf $\mathcal{O}_{\Delta}$ of the diagonal, we obtain an isomorphism

$$
\mathcal{K}^{\bullet} \simeq \mathbf{R} \mathcal{H o m}_{\mathcal{O}_{X \times Y}}^{\bullet}\left(\mathbf{R} \mathcal{H o m}_{\mathcal{O}_{X \times Y}}^{\bullet}\left(\mathcal{K} \cdot, \pi_{Y}^{!} \mathcal{O}_{Y}\right), \pi_{X}^{!} \mathcal{O}_{X}\right) .
$$

Since $\mathbf{R} \operatorname{Hom}_{X \times Y}^{\bullet}\left(\mathcal{K}^{\bullet}, \pi_{Y}^{!} \mathcal{O}_{Y}\right)$ is of finite homological dimension over $X$, we conclude by Proposition 2.11

Remark 4.3. When $X$ and $Y$ are smooth, Proposition 4.2 is equivalent to the fact that any equivalence commutes with the Serre functors. Thus, Proposition $4.2 \mathrm{can}$ be considered as a generalisation of that property to arbitrary singular schemes.

Our next aim is to prove that any integral Fourier-Mukai partner of a projective Cohen-Macaulay (resp. Gorenstein) scheme $X$ is also Cohen-Macaulay (resp. Gorenstein).

Theorem 4.4. Let $X$ be a projective equidimensional Cohen-Macaulay scheme and $Y$ a projective Fourier-Mukai partner of $X$.

(1) If $Y$ is reduced, then $Y$ is equidimensional of dimension $m=\operatorname{dim} X$.

(2) If $Y$ is equidimensional and $\operatorname{dim} Y=\operatorname{dim} X$, then $Y$ is Cohen-Macaulay. Moreover, if $X$ is Gorenstein, then $Y$ is Gorenstein as well.

Proof. By Proposition 4.2 the integral functors

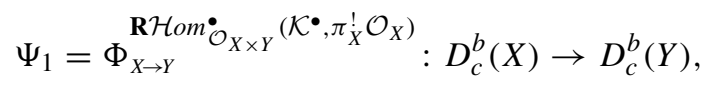

$$
\begin{aligned}
& \left.\Psi_{2}=\Phi_{X \rightarrow Y}^{\mathbf{R} \mathcal{H}_{X} m_{\mathcal{O}_{X \times Y}}} \mathcal{K}^{\bullet}, \pi_{\dot{Y}}^{!} \mathcal{O}_{Y}\right): D_{c}^{b}(X) \rightarrow D_{c}^{b}(Y)
\end{aligned}
$$


are naturally isomorphic. Hence, for any 1.c.i zero cycle $Z_{x}$ supported on a closed point $x \in X$ and any closed point $y \in Y$ we have

$$
\mathbf{R} \mathcal{H o m}_{\mathcal{O}_{Y}}^{\cdot}\left(\mathcal{O}_{y}, \Psi_{1}\left(\mathcal{O}_{Z_{x}}\right)\right) \simeq \mathbf{R} \mathcal{H o m}_{\mathcal{O}_{Y}}^{\cdot}\left(\mathcal{O}_{y}, \Psi_{2}\left(\mathcal{O}_{Z_{x}}\right)\right) .
$$

This gives rise to the formula

$$
\mathbf{R} \mathcal{H o m}_{\mathcal{O}_{Y}}^{\cdot}\left(\Phi_{Z_{x}}\left(\mathcal{D}_{Z_{x}}^{\bullet}\right), \mathcal{O}_{y}\right) \simeq \mathbf{R} \mathcal{H o m}_{\mathcal{O}_{Y}}^{\bullet}\left(\Phi_{Z_{x}}\left(\mathcal{O}_{Z_{x}}\right), \mathbf{R} \mathcal{H o m}_{\mathcal{O}_{Y}}^{\bullet}\left(\mathcal{O}_{y}, \mathcal{O}_{Y}\right)\right)[m],
$$

where $\Phi_{Z_{x}}: D_{c}^{b}\left(Z_{x}\right) \rightarrow D_{c}^{b}(Y)$ is the integral functor of the kernel $\mathcal{K}_{Z_{x}}=\mathbf{L} j_{Z_{x}}^{*} \mathcal{K} \cdot \epsilon$ $D_{c}^{b}\left(Z_{x} \times Y\right)$ (see Lemmas 4.5 and 4.6 for details).

Since $X$ is Cohen-Macaulay, every 1.c.i. cycle $Z_{x}$ is Cohen-Macaulay as well, and its dualising complex $\mathcal{D}_{Z_{x}}^{\cdot}$ is a single sheaf $\omega_{Z_{x}}$.

As $\Phi$ is an equivalence of categories, it follows from Lemma 3.5 that the objects $\Phi_{Z_{x}}\left(\mathcal{O}_{Z_{x}}\right) \simeq \Phi\left(\mathcal{O}_{Z_{x}}\right)$ for all 1.c.i. cycles $Z_{x}$ form a spanning class for $D_{c}^{b}(Y)$. Thus, if we fix a closed point $y \in Y$, there is a l.c.i. cycle $Z_{x}$ such that $y \in \operatorname{supp}\left(\Phi_{Z_{x}}\left(\mathcal{O}_{Z_{x}}\right)\right)$.

Let us denote by $\phi: Y^{\prime}=\operatorname{Spec} \mathcal{O}_{Y, y} \rightarrow Y$ the natural flat morphism. The composition $\Phi_{Z_{x}}^{\prime}=\phi^{*} \circ \Phi_{Z_{x}}$ is an integral functor with kernel $\mathcal{K}_{Z_{x}}^{\prime}=(1 \times \phi)^{*} \mathcal{K}_{Z_{x}}^{\bullet} \in D_{c}^{b}\left(Z_{x} \times Y^{\prime}\right)$. If we apply $\phi^{*}$ to (4.1) we get the analogous formula

$$
\mathbf{R} \mathcal{H o m}_{\mathcal{O}_{Y^{\prime}}}^{\cdot}\left(\Phi_{Z_{x}}^{\prime}\left(\omega_{Z_{x}}\right), \mathcal{O}_{y}\right) \simeq \mathbf{R} \mathcal{H o m}_{\mathcal{O}_{Y^{\prime}}}\left(\Phi_{Z_{x}}^{\prime}\left(\mathcal{O}_{Z_{x}}\right), \mathbf{R} \mathcal{H o m}_{\mathcal{O}_{Y^{\prime}}}\left(\mathcal{O}_{y}, \mathcal{O}_{Y^{\prime}}\right)\right)[m]
$$

After shifting degrees if necessary, we can assume that $\mathcal{H}^{0}\left(\mathcal{K}^{\prime \bullet}{ }_{Z_{x}}\right) \neq 0$ and $\mathcal{H}^{i}\left(\mathcal{K}^{\prime \bullet} Z_{x}\right)$ $=0$ for $i>0$. Then $\mathcal{H}^{0}\left(\Phi_{Z_{x}}^{\prime}\left(\mathcal{O}_{Z_{x}}\right)\right) \neq 0$ and $\mathcal{H}^{i}\left(\Phi_{Z_{x}}^{\prime}\left(\mathcal{O}_{Z_{x}}\right)\right)=0$ for $i>0$. For every

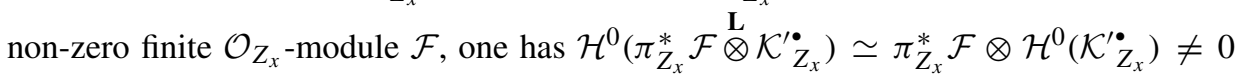
and $\mathcal{H}^{i}\left(\pi_{Z_{x}}^{*} \mathcal{F} \stackrel{\mathbf{L}}{\otimes} \mathcal{K}_{Z_{x}}^{\prime}{ }_{Z_{x}}\right)=0$ for $i>0$, where $\pi_{Z_{x}}: Z_{x} \times Y^{\prime} \rightarrow Z_{x}$ is the projection. Thus, $\mathcal{H}^{0}\left(\Phi_{Z_{x}}^{\prime}(\mathcal{F})\right) \neq 0$ and $\mathcal{H}^{i}\left(\Phi_{Z_{x}}^{\prime}(\mathcal{F})\right)=0$ for $i>0$.

It follows that $\mathbf{R} \mathcal{H o m}_{\mathcal{O}_{Y^{\prime}}}\left(\Phi_{Z_{x}}^{\prime}\left(\omega_{Z_{x}}\right), \mathcal{O}_{y}\right)$ has no negative cohomology sheaves and that its 0th cohomology sheaf is non-zero. By [4.2), the same happens for the object $\mathcal{Q}^{\bullet}=\mathbf{R} \mathcal{H o m}_{\mathcal{O}_{Y^{\prime}}}^{\cdot}\left(\Phi_{Z_{x}}^{\prime}\left(\mathcal{O}_{Z_{x}}\right), \mathbf{R} \mathcal{H}_{o m} \dot{\mathcal{O}}_{Y^{\prime}}\left(\mathcal{O}_{y}, \mathcal{O}_{Y^{\prime}}\right)\right)[m]$, that is,

$$
\mathcal{H}^{i}\left(\mathcal{Q}^{\bullet}\right)=0 \quad \text { for } i<0, \quad \text { and } \quad \mathcal{H}^{0}\left(\mathcal{Q}^{\bullet}\right) \neq 0 .
$$

(1) Assume that $Y$ is reduced and we choose $y$ to be a smooth point of an irreducible component $Y_{0}$ of $Y$. Then $\mathbf{R} \mathcal{H o m}_{\mathcal{O}_{Y^{\prime}}}\left(\mathcal{O}_{y}, \mathcal{O}_{Y^{\prime}}\right) \simeq \mathcal{O}_{y}[-n]$, where $n=\operatorname{dim} Y_{0}$, and $\mathcal{Q}^{\bullet} \simeq \mathbf{R} \mathcal{H o m}_{\mathcal{O}_{Y^{\prime}}}\left(\Phi_{Z_{x}}^{\prime}\left(\mathcal{O}_{Z_{x}}\right), \mathcal{O}_{y}\right)[m-n]$. Since both $\mathcal{Q}^{\bullet}$ and $\mathbf{R} \mathcal{H}_{\text {om }} \dot{\mathcal{O}}_{Y^{\prime}}\left(\Phi_{Z_{x}}^{\prime}\left(\mathcal{O}_{Z_{x}}\right), \mathcal{O}_{y}\right)$ have no negative cohomology sheaves and non-zero 0th cohomology sheaf, one must have $m-n=0$. Thus all the irreducible components of $Y$ have dimension $m$.

(2) Let $y \in Y$ be a closed point and let $j_{0}$ be the first index $j$ with $\mathcal{E}_{x} t_{\mathcal{O}_{Y^{\prime}}}^{j}\left(\mathcal{O}_{y}, \mathcal{O}_{Y^{\prime}}\right)$ $\neq 0$, that is, $j_{0}$ is the depth of the local ring $\mathcal{O}_{Y, y}$. Then

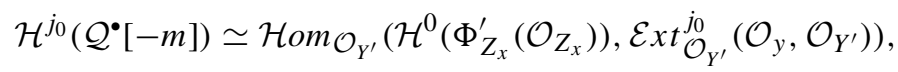


which is not zero because $\mathcal{E} x t_{\mathcal{O}_{Y^{\prime}}}^{j_{0}}\left(\mathcal{O}_{y}, \mathcal{O}_{Y^{\prime}}\right)$ is a non-zero $\mathcal{O}_{y}$-vector space. Hence $j_{0} \geq$ $m=\operatorname{dim} Y$ (cf. (4.3)), and $Y$ is Cohen-Macaulay.

Assume now that $X$ is Gorenstein. Then $\omega_{Z_{x}} \simeq \mathcal{O}_{Z_{x}}$ and we have

$$
\mathbf{R} \mathcal{H o m}_{\mathcal{O}_{Y^{\prime}}}^{\cdot}\left(\Phi_{Z_{x}}^{\prime}\left(\mathcal{O}_{Z_{x}}\right), \mathcal{O}_{y}\right) \simeq \mathbf{R} \mathcal{H o m}_{\mathcal{O}_{Y^{\prime}}}^{\cdot}\left(\Phi_{Z_{x}}^{\prime}\left(\mathcal{O}_{Z_{x}}\right), \mathbf{R} \mathcal{H o m}_{\mathcal{O}_{Y^{\prime}}}^{\cdot}\left(\mathcal{O}_{y}, \mathcal{O}_{Y^{\prime}}\right)\right)[m],
$$

by 4.2. Since $\mathcal{E} x t_{\mathcal{O}_{Y^{\prime}}}^{j}\left(\mathcal{O}_{y}, \mathcal{O}_{Y^{\prime}}\right)=0$ for $j<m$, we deduce that

$$
\operatorname{Hom}_{Y^{\prime}}\left(\mathcal{H}^{0}\left(\Phi_{Z_{x}}^{\prime}\left(\mathcal{O}_{Z_{x}}\right)\right), \mathcal{O}_{y}\right) \simeq \operatorname{Hom}_{Y^{\prime}}\left(\mathcal{H}^{0}\left(\Phi_{Z_{x}}^{\prime}\left(\mathcal{O}_{Z_{x}}\right)\right), \mathcal{E} x t_{\mathcal{O}_{Y^{\prime}}}^{m}\left(\mathcal{O}_{y}, \mathcal{O}_{Y^{\prime}}\right)\right),
$$

and

$\operatorname{Hom}_{\mathcal{O}_{y}}\left(\mathcal{H}^{0}\left(\Phi_{Z_{x}}^{\prime}\left(\mathcal{O}_{Z_{x}}\right)\right) \otimes \mathcal{O}_{y}, \mathcal{O}_{y}\right) \simeq \operatorname{Hom}_{\mathcal{O}_{y}}\left(\mathcal{H}^{0}\left(\Phi_{Z_{x}}^{\prime}\left(\mathcal{O}_{Z_{x}}\right)\right) \otimes \mathcal{O}_{y}, \mathcal{E}_{x} t_{\mathcal{O}_{Y^{\prime}}}^{m}\left(\mathcal{O}_{y}, \mathcal{O}_{Y^{\prime}}\right)\right)$.

Thus, $\operatorname{dim} \mathcal{E} t_{\mathcal{O}_{Y^{\prime}}}^{m}\left(\mathcal{O}_{y}, \mathcal{O}_{Y^{\prime}}\right)=1$ and $Y$ is Gorenstein.

We now prove the auxiliary lemmas used in the proof of Theorem 4.4

Lemma 4.5. R $\mathcal{H o m}_{\mathcal{O}_{Y}}\left(\mathcal{O}_{y}, \Psi_{1}\left(\mathcal{O}_{Z_{x}}\right)\right) \simeq \mathbf{R} \mathcal{H o m}_{\mathcal{O}_{Y}}^{\bullet}\left(\Phi_{Z_{x}}\left(\mathcal{D}_{Z_{x}}^{\bullet}\right), \mathcal{O}_{y}\right)$.

Proof. We first compute $\Psi_{1}\left(\mathcal{O}_{Z_{x}}\right)$. By Corollary 2.12 and Lemma 2.13.

$$
\Psi_{1}\left(\mathcal{O}_{Z_{x}}\right) \simeq \Psi_{1}\left(\mathcal{O}_{Z_{x}}^{\#}\right) \simeq\left(\Phi\left(\mathcal{O}_{Z_{x}}^{\sharp}\right)\right)^{\sharp} \simeq\left(\Phi_{Z_{x}}\left(\mathcal{D}_{Z_{x}}^{\cdot}\right)\right)^{\sharp} .
$$

where the last isomorphism is due to the isomorphism $\mathcal{O}_{Z_{x}}^{\sharp} \simeq j_{Z_{x} *} \mathcal{D}_{Z_{x}}^{\cdot}$, which follows by relative duality for the closed immersion $j_{Z_{x}}: Z_{x} \hookrightarrow X$. Considering (1.1), we obtain

$$
\begin{aligned}
& \mathbf{R} \mathcal{H o m}_{\mathcal{O}_{Y}}^{\bullet}\left(\mathcal{O}_{y}, \Psi_{1}\left(\mathcal{O}_{Z_{x}}\right)\right) \simeq \mathbf{R} \mathcal{H o m}_{\mathcal{O}_{Y}}^{\cdot}\left(\mathcal{O}_{y},\left(\Phi_{Z_{x}}\left(\mathcal{D}_{Z_{x}}^{\cdot}\right)\right)^{\sharp}\right) \\
& \simeq \mathbf{R} \mathcal{H o m}_{\mathcal{O}_{Y}}^{\cdot}\left(\mathcal{O}_{y} \stackrel{\mathbf{L}}{\otimes} \Phi_{Z_{x}}\left(\mathcal{D}_{Z_{x}}^{\cdot}\right), \mathcal{D}_{Y}^{\cdot}\right) \simeq \mathbf{R} \mathcal{H o m}_{\mathcal{O}_{Y}}^{\cdot}\left(\Phi_{Z_{x}}\left(\mathcal{D}_{Z_{x}}^{\cdot}\right), \mathcal{O}_{y}^{\sharp}\right) \text {. }
\end{aligned}
$$

Relative duality for the closed immersion $\{y\} \hookrightarrow Y$ gives $\mathcal{O}_{y}^{\sharp} \simeq \mathcal{O}_{y}$, which finishes the proof.

Lemma 4.6. One has

$$
\mathbf{R} \mathcal{H o m}_{\mathcal{O}_{Y}}^{\cdot}\left(\mathcal{O}_{y}, \Psi_{2}\left(\mathcal{O}_{Z_{x}}\right)\right) \simeq \mathbf{R} \mathcal{H o m}_{\mathcal{O}_{Y}}^{\cdot}\left(\Phi_{Z_{x}}\left(\mathcal{O}_{Z_{x}}\right), \mathbf{R} \mathcal{H o m}_{\mathcal{O}_{Y}}^{\cdot}\left(\mathcal{O}_{y}, \mathcal{O}_{Y}\right)\right)[m] .
$$

Proof. We first compute $\Psi_{2}\left(\mathcal{O}_{Z_{x}}\right)$. Let $\pi_{Z_{x}}$ and $\bar{\pi}_{Y}$ denote the projections of $Z_{x} \times Y$ onto $Z_{x}$ and $Y$ respectively. Using the fact that $\mathcal{O}_{Z_{x} \times Y}$ is of finite homological dimension as a module over $\mathcal{O}_{X \times Y}$ and taking into account $(1.2)$, we obtain

$$
\Psi_{2}\left(\mathcal{O}_{Z_{x}}\right) \simeq \bar{\pi}_{Y *} \mathbf{R} \mathcal{H o m}_{\mathcal{O}_{Z_{x} \times Y}}\left(\mathcal{K}_{Z_{x}}^{\bullet}, \mathbf{L} j_{Z_{x}}^{*} \pi_{Y}^{!} \mathcal{O}_{Y}\right) .
$$

The term $\mathbf{L} j_{Z_{x}}^{*} \pi_{Y}^{!} \mathcal{O}_{Y}$ is computed as $\mathbf{L} j_{Z_{x}}^{*} \pi_{X}^{*} \mathcal{D}_{X}^{*} \simeq \pi_{Z_{X}}^{*} \mathbf{L} j_{Z_{X}}^{*} \mathcal{D}_{X}^{*}$. Furthermore, relative duality for the regular immersion $j_{Z_{x}}: Z_{x} \hookrightarrow X$ gives $j_{Z_{x}}^{!} \mathcal{O}_{X} \simeq \mathcal{O}_{Z_{x}}[-m]$, where $m=$ 
$\operatorname{dim} X$ and $\mathcal{D}_{Z_{x}}^{\bullet} \simeq j_{Z_{x}}^{!} \mathcal{O}_{X} \stackrel{\mathbf{L}}{\otimes} \mathbf{L} j_{Z_{x}}^{*} \mathcal{D}_{X}^{\bullet}$. Thus, $\pi_{Z_{x}}^{*} \mathbf{L}_{Z_{x}}^{*} \mathcal{D}_{X}^{\bullet} \simeq \pi_{Z_{x}}^{*} \mathcal{D}_{Z_{x}}^{\bullet}[m] \simeq \bar{\pi}_{Y}^{!} \mathcal{O}_{Y}[m]$, so that

$$
\begin{aligned}
\Psi_{2}\left(\mathcal{O}_{Z_{x}}\right) & \simeq \bar{\pi}_{Y *} \mathbf{R} \mathcal{H o m}_{\mathcal{O}_{Z_{x} \times Y}}\left(\mathcal{K}_{Z_{x}}^{\bullet}, \bar{\pi}_{Y}^{!} \mathcal{O}_{Y}[m]\right) \\
& \simeq \mathbf{R} \mathcal{H o m}_{\mathcal{O}_{Y}}\left(\Phi_{Z_{x}}\left(\mathcal{O}_{Z_{x}}\right), \mathcal{O}_{Y}[m]\right) .
\end{aligned}
$$

Then

$$
\mathbf{R} \mathcal{H o m}_{\mathcal{O}_{Y}}^{\bullet}\left(\mathcal{O}_{y}, \Psi_{2}\left(\mathcal{O}_{Z_{x}}\right)\right) \simeq \mathbf{R H o m}_{\mathcal{O}_{Y}}^{\bullet}\left(\mathcal{O}_{y}, \mathbf{R H o m}_{\mathcal{O}_{Y}}^{\bullet}\left(\Phi_{Z_{x}}\left(\mathcal{O}_{Z_{x}}\right), \mathcal{O}_{Y}[m]\right)\right)
$$

and one concludes by (1.1).

Acknowledgments. We would like to thank the authors of the forthcoming book [2] for sharing with us their notes and to the anonymous referees for comments and suggestions which helped us to improve the manuscript. Ana Cristina López Martín would also like to thank Miles Reid for useful comments and for his warm welcome in Warwick.

Work supported by research projects MTM2006-04779 (MEC) and SA001A07 (JCYL).

\section{References}

[1] Abramovich, D., Chen, J.-C.: Flops, flips and perverse point sheaves on threefold stacks. J. Algebra 290, 372-407 (2005) Zbl 1087.14010 MR 2153260

[2] Bartocci, C., Bruzzo, U., Hernández Ruipérez, D.: Fourier-Mukai and Nahm Transforms in Geometry and Mathematical Physics. Progr. Math., Birkhäuser (2008), to appear

[3] Bartocci, C., Bruzzo, U., Hernández Ruipérez, D., Muñoz Porras, J. M.: Relatively stable bundles over elliptic fibrations. Math. Nachr. 238, 23-36 (2002) Zbl 1033.14007 MR 2100050

[4] Bökstedt, M., Neeman, A.: Homotopy limits in triangulated categories. Compos. Math. 86, 209-234 (1993) Zbl 0802.18008 MR 1214458

[5] Bondal, A., Orlov, D.: Derived categories of coherent sheaves. In: Proc. Int. Congress Math., Vol. II (Beijing, 2002), Higher Ed. Press, Beijing, 47-56 (2002) Zbl 0996.18007 MR 1957019

[6] Bondal, A. I., Orlov, D. O.: Semi orthogonal decomposition for algebraic varieties. MPIM Preprint 95/15 (1995), math.AG/9506012

[7] Bridgeland, T.: Equivalences of triangulated categories and Fourier-Mukai transforms. Bull. London Math. Soc. 31, 25-34 (1999) Zbl 0937.18012 MR 1651025

[8] Bridgeland, T.: Flops and derived categories. Invent. Math. 147, 613-632 (2002) Zbl 1085.14017 MR 1893007

[9] Burban, I., Kreußler, B.: Fourier-Mukai transforms and semistable sheaves on nodal Weierstraß cubics. J. Reine Angew. Math. 564, 45-82 (2005) Zbl 1085.14018 MR 2155085

[10] Burban, I., Kreußler, B.: Derived categories of irreducible projective curves of arithmetic genus one. Compos. Math. 142, 1231-1262 (2006) Zbl 1103.14007 MR 2264663

[11] Burban, I., Kreußler, B.: On a relative Fourier-Mukai transform on genus one fibrations. Manuscripta Math. 120, 283-306 (2006) Zbl 1105.18011 MR 2243564

[12] Catanese, F., Franciosi, M., Hulek, K., Reid, M.: Embeddings of curves and surfaces. Nagoya Math. J. 154, 185-220 (1999) Zbl 0933.14003 MR 1689180 
[13] Chen, J.-C.: Flops and equivalences of derived categories for threefolds with only terminal Gorenstein singularities. J. Differential Geom. 61, 227-261 (2002) Zbl 1090.14003 MR 1972146

[14] Hartshorne, R.: Residues and Duality (with an appendix by P. Deligne). Lecture Notes in Math. 20, Springer, Berlin (1966) Zbl 0212.26101 MR 0222093

[15] Hein, G., Ploog, D.: Fourier-Mukai transforms and stable bundles on elliptic curves. Beiträge Algebra Geom. 46, 423-434 (2005) Zbl 1093.14047 MR 2196927

[16] Hernández Ruipérez, D., López Martín, A. C., Sancho de Salas, F.: Fourier-Mukai transform for Gorenstein schemes. Adv. Math. 211, 594-620 (2007) Zbl 1118.14022 MR 2323539

[17] Illusie, L.: Frobenius et dégénérescence de Hodge. In: Introduction à la théorie de Hodge, Panor. Synthèses 3, Soc. Math. France, Paris, 113-168 (1996) MR 1409820

[18] Kawamata, Y.: $D$-equivalence and $K$-equivalence. J. Differential Geom. 61, 147-171 (2002) Zbl 1056.14021 MR 1949787

[19] Kawamata, Y.: Francia's flip and derived categories. In: Algebraic Geometry, de Gruyter, Berlin, 197-215 (2002) Zbl 1092.14023 MR 1954065

[20] Kodaira, K.: On compact analytic surfaces. II, III. Ann. of Math. (2) 77, 563-626 (1963); ibid. 78, 1-40 (1963) Zbl 0118.15802 MR 0184257

[21] Kollár, J., Mori, S.: Birational Geometry of Algebraic Varieties. Cambridge Tracts Math. 134, Cambridge Univ. Press, Cambridge (1998) Zbl 0926.14003 MR 1658959

[22] Kuznetsov, A. G., Hyperplane sections and derived categories. Izv. Ross. Akad. Nauk Ser. Mat. 70, 23-128 (2006) Zbl 1133.14016 MR 2238172

[23] Miranda, R.: Smooth models for elliptic threefolds. In: The Birational Geometry of Degenerations (Cambridge, MA, 1981), Progr. Math. 29, Birkhäuser Boston, MA, 85-133 (1983) Zbl 0583.14014 MR 0690264

[24] Neeman, A.: The Grothendieck duality theorem via Bousfield's techniques and Brown representability. J. Amer. Math. Soc. 9, 205-236 (1996) Zbl 0864.14008 MR 1308405

[25] Orlov, D. O., Equivalences of derived categories and $K 3$ surfaces. J. Math. Sci. (New York) 84, 1361-1381 (1997) Zbl 0938.14019 MR 1465519

[26] Seidel, P., Thomas, R.: Braid group actions on derived categories of coherent sheaves. Duke Math. J. 108, 37-108 (2001) Zbl 1092.14025 MR 1831820

[27] Spaltenstein, N.: Resolutions of unbounded complexes. Compos. Math. 65, 121-154 (1988) Zbl 0636.18006 MR 0932640 